\title{
DEEP REINFORCEMENT LEARNING BASED ACTIVE QUEUE MANAGEMENT FOR IOT NETWORKS
}

\author{
by \\ Minsu Kim \\ B.Eng., Yonsei University, the Republic of Korea, 2017 \\ A Thesis \\ Presented to Ryerson University \\ in partial fulfilment of the \\ requirements for the degree of \\ Master of Applied Science \\ in the program of \\ Computer Networks
}

Toronto, Ontario, Canada, 2019

(C)Minsu Kim 2019 


\section{Author's Declaration}

I hereby declare that I am the sole author of this dissertation. This is a true copy of the dissertation, including any required final revisions, as accepted by my examiners.

I authorize Ryerson University to lend this dissertation to other institutions or individuals for the purpose of scholarly research.

I further authorize Ryerson University to reproduce this dissertation by photocopying or by other means, in total or in part, at the request of other institutions or individuals for the purpose of scholarly research.

I understand that my dissertation may be made electronically available to the public. 


\title{
Abstract \\ Deep Reinforcement Learning based Active Queue Management for IoT Networks
}

(C)Minsu Kim, 2019

\author{
Master of Applied Science \\ Computer Networks \\ Ryerson University
}

Internet of Things (IoT) has pervaded most aspects of our life through the Fourth Industrial Revolution. It is expected that a typical family home could contain several hundreds of smart devices by 2022. Current network architecture has been moving to fog/edge architecture to have the capacity for IoT. However, in order to deal with the enormous amount of traffic generated by those devices and reduce queuing delay, novel self-learning network management algorithms are required on fog/edge nodes. For efficient network management, Active Queue Management (AQM) has been proposed which is the intelligent queuing discipline. In this paper, we propose a new AQM based on Deep Reinforcement Learning (DRL) to handle the latency as well as the trade-off between queuing delay and throughput. We choose Deep Q-Network (DQN) as a baseline of our scheme, and compare our approach with various AQM schemes by deploying them on the interface of fog/edge node in IoT infrastructure. We simulate the AQM schemes on the different bandwidth and round trip time (RTT) settings, and in the empirical results, our approach outperforms other AQM schemes in terms of delay and jitter maintaining above-average throughput and verifies that DRL applied AQM is an efficient network manager for congestion. 


\section{Acknowledgements}

I would like to express my sincere gratitude to my supervisor Prof. Alagan Anpalagan for giving me lots of opportunities and support, from leading me into Canada to completing my studies with meaningful results. Without his trust, help and guidance, I would not have completed my master's studies.

Also I would like to extend my gratitude to the Computer Networks Program and the School of Graduate Studies at Ryerson University which provided me with a chance to pursue my master's degree and all kinds of support for my studies.

I am grateful to Prof. Muhammad Jaseemuddin, Bobby Ma, and Javad Alirezaie for being members of my defense committee and giving me constructive advice for a more solid thesis.

It was my pleasure to be a member of WINCORE Lab and spend time with great colleagues and fellows, and I have felt their warm hearts and a friendly environment.

In addition, I would like to thank to my family for their trust and dedication during my master's studies, and my friends from all communities for their pray and encouragement.

Last but certainly not least, my special thanks go to my significant other, Buon, who is always a constant source of love and motivation for my life. 


\section{Table of Contents}

Author's Declaration ii

Abstract $\quad$ iii

Acknowledgements $\quad$ iv

List of Tables $\quad$ viii

List of Figures $\quad$ ix

List of Abbreviations $\quad$ xi

1 Introduction $\quad 1$

1.1 Background . . . . . . . . . . . . . . . . . . . . 1

1.1.1 Fog/Edge Computing Architecture for Internet of Things . . . . . . . 1

1.1.2 Active Queue Management for Internet of Things . . . . . . . . . . . 3

1.1.3 Reinforcement Learning for Network Management . . . . . . . . . . . 3

1.2 Motivation and Objective ...................... 4

1.3 Thesis Contributions . . . . . . . . . . . . . . . . . 4

1.4 Thesis Organization . . . . . . . . . . . . . . . . . . . 5

2 Literature Review of Active Queue Management $\quad 6$

2.1 Active Queue Management Schemes . . . . . . . . . . . . . . . 7 
2.1.1 Random Early Detection (RED) . . . . . . . . . . . . 7

2.1.2 Controlled Delay (CoDel) . . . . . . . . . . . . . . 7

2.1.3 Proportional Integral controller Enhanced (PIE) . . . . . . . . . . 8

2.2 Machine Learning Applied AQM and Network Traffic Scheduling Algorithms 9

2.3 Traffic Management for IoT Applications . . . . . . . . . . . . . . . . 10

2.4 Summary . . . . . . . . . . . . . . . . . . . . . 12

3 System Model $\quad 14$

3.1 Deep Q-Network . . . . . . . . . . . . . . . . . . . 14

3.2 DQN based AQM System Design . . . . . . . . . . . . . . . 17

3.2.1 Process of Selecting Action . . . . . . . . . . . . . . 17

3.2.2 Reward Engineering . . . . . . . . . . . . . . . 18

3.2.3 Training Process . . . . . . . . . . . . . . . . . . . . 20

3.3 Summary . . . . . . . . . . . . . . . . . . . 20

4 Experiment Setup $\quad 23$

4.1 Characteristics of IoT devices . . . . . . . . . . . . . . . 23

4.2 IoT Testbed . . . . . . . . . . . . . . . . . . . . . . . . 24

4.3 Assumptions and Parameter Setting . . . . . . . . . . . . . . . . . . . . 24

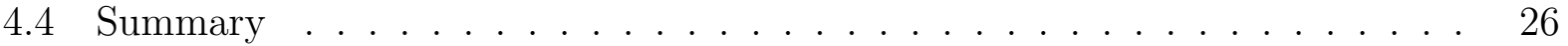

5 Results and Analysis $\quad 29$

5.1 Comparison on Queuing Delay and Occupancy . . . . . . . . . . . . . . . 29

5.2 Comparison on Different Bandwidth . . . . . . . . . . . . . . . 30

5.3 Comparison on Different Baseline RTT . . . . . . . . . . . . . . . . . 31

5.4 Analysis on Different IoT Categories . . . . . . . . . . . . . . . 33

5.4 .1 UDP based IoT flows . . . . . . . . . . . . . . . . 35

5.4 TCP based IoT flows . . . . . . . . . . . . . . . . . 35

5.5 Analysis on Reward Trajectory for Model Optimization . . . . . . . . . . . 37 
5.6 Summary . . . . . . . . . . . . . . . . . . . . . 40

6 Conclusion $\quad 41$

6.1 Conclusion and Summary . . . . . . . . . . . . . . . . 41

6.2 Future Work . . . . . . . . . . . . . . . . . . 42

6.2.1 Energy Efficient AQM Design . . . . . . . . . . . . . . . 42

6.2.2 Construction of IoT-realistic Simulation Environment . . . . . . . . . 42

A Dequeue Rate Calculation in PIE 43

B AQM Performance Comparison in Various Environments 44

$\begin{array}{ll}\text { Bibliography } & 47\end{array}$ 


\section{List of Tables}

2.1 Summary of related works. . . . . . . . . . . . . . . . . . . 13

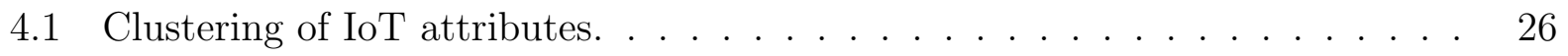

4.2 Measure of attribute clusters. . . . . . . . . . . . . . . . 27

4.3 Environmental setup parameters. . . . . . . . . . . . . . . . 27

4.4 Comparison AQM setup parameters. . . . . . . . . . . . . . . . 28

4.5 DQN based AQM algorithm setup parameters. . . . . . . . . . . 28

5.1 Reward scaling factor sensitivity; P2P link data rate = 1Mbps; Baseline RTT $=0 \mathrm{~ms} ;$ Number of IoT devices $=63 \ldots \ldots \ldots \ldots$ 


\section{List of Figures}

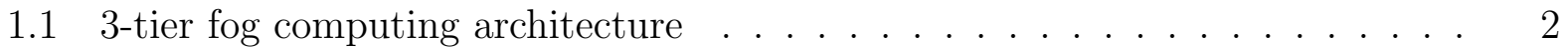

3.1 Markov Decision Process. . . . . . . . . . . . . . . . . . . . . 15

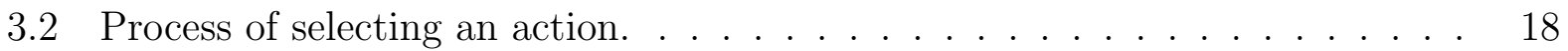

3.3 Flowchart of DQN based AQM training . . . . . . . . . . . . . . 21

4.1 Overview of the IoT testbed $\ldots \ldots \ldots \ldots \ldots$

5.1 Empirical CDF of queuing delay for flows of IoT devices; P2P link data rate $=1 \mathrm{Mbps} ;$ Baseline RTT $=0 \mathrm{~ms}$; Reward scaling factor $\delta=0.5 \ldots \ldots$

5.2 Comparison of queue occupancy of AQM schemes; P2P link data rate = 1Mbps; Baseline RTT $=0 \mathrm{~ms}$; Number of IoT devices $=21$; Reward scaling

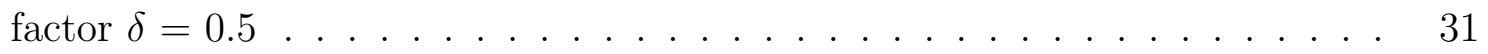

5.3 Throughput per flows of 21 IoT devices; Baseline RTT = 0ms; Reward scaling

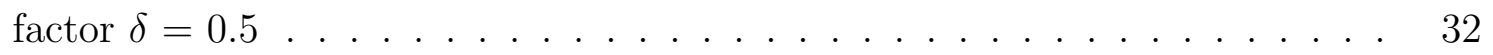

5.4 Mean delay per flows of 21 IoT devices; Baseline RTT = 0ms; Reward scaling

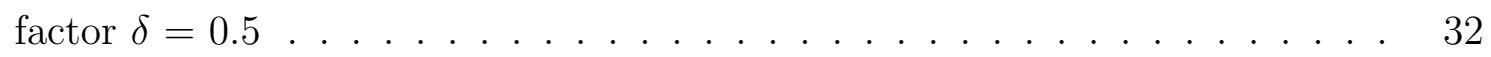

5.5 Throughput per flows of 63 IoT devices; P2P link data rate = 1Mbps; Reward scaling factor $\delta=0.5 \ldots \ldots \ldots \ldots \ldots \ldots \ldots \ldots \ldots$

5.6 Mean delay per flows of 63 IoT devices; P2P link data rate $=1 \mathrm{Mbps}$; Reward scaling factor $\delta=0.5 \ldots \ldots \ldots \ldots \ldots \ldots \ldots$ 
5.7 Maximum delay per flows of 63 IoT devices; P2P link data rate $=1 \mathrm{Mbps}$; Reward scaling factor $\delta=0.5 \ldots \ldots . \ldots . \ldots . \ldots . \ldots 34$

5.8 Most frequent delay per flows of 63 IoT devices; P2P link data rate $=1 \mathrm{Mbps}$; Reward scaling factor $\delta=0.5 \ldots \ldots$. . . . . . . . . . . . . . 35

5.9 Throughput per UDP flows of 18 IoT devices; P2P link data rate $=1 \mathrm{Mbps}$;

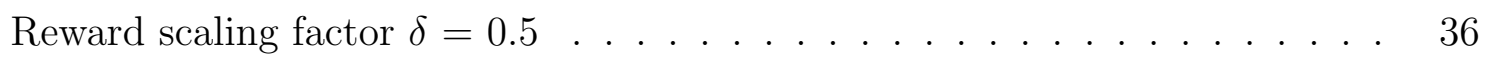

5.10 Mean Delay per UDP flows of 18 IoT devices; P2P link data rate $=1 \mathrm{Mbps}$; Reward scaling factor $\delta=0.5 \ldots \ldots$. . . . . . . . . . . 36

5.11 Mean Jitter per UDP flows of 18 IoT devices; P2P link data rate $=1 \mathrm{Mbps}$;

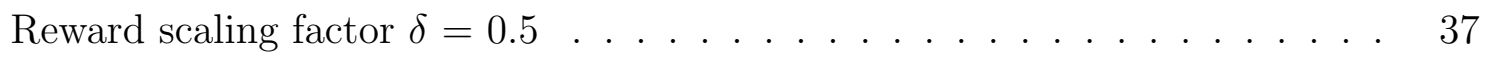

5.12 Throughput per TCP flows of 45 IoT devices; P2P link data rate $=1 \mathrm{Mbps}$; Reward scaling factor $\delta=0.5 \ldots \ldots . \ldots . \ldots . \ldots . \ldots$

5.13 Mean Delay per TCP flows of 45 IoT devices; P2P link data rate $=1 \mathrm{Mbps}$; Reward scaling factor $\delta=0.5 \ldots \ldots$. . . . . . . . . . . . . 38

5.14 Comparison of reward on non-trained/trained model; P2P link data rate = 1Mbps; Baseline RTT $=0 \mathrm{~ms} ;$ Number of IoT devices $=63$; Reward scaling factor $\delta=0.5 \ldots \ldots \ldots \ldots \ldots \ldots$ 


\section{List of Abbreviations}

6LoWPAN IPv6 over Low-power wireless personal area network

APs Access Points

AQM Active Queue Management

CDF Cumulative distribution function

CoDel Controlled Delay

CSMA Carrier-sense Multiple Access

DL Deep Learning

DNN Deep Neural Network

DQN Deep Q-Network

DRL Deep Reinforcement Learning

FAN Fog access node

FIFO First-In-First-Out

FQ-CoDel FlowQueue-Controlled Delay

HTTPS Hyper Text Transfer Protocol Secure

HVFT High Volume Flexible Time

IETF Internet Engineering Task Force

IoT Internet of Things

MDP Markov decision process

ML Machine Learning

MLP Multilayer Perceptron

P2P peer-to-peer

P-FIFO Priority-FIFO-fast

PIE Proportional Integral controller Enhanced 


\author{
QoS Quality of Service \\ RED Random Early Detection \\ RL Reinforcement Learning \\ RTT Round Trip Time \\ SDN Software Defined Network \\ TCP Transmission Control Protocol \\ TD Temporal Difference \\ UDP User Datagram Protocol
}




\section{Chapter 1}

\section{Introduction}

\subsection{Background}

The concept and definition of Internet of Things (IoT) is derived from a connectivity of every machine device on the accelerative convergence of many technologies such as wireless technologies, embedded systems, big data analysis, and real-time processing for smart automation toward future Internet. International Data Corporation (IDC) expects that worldwide IoT market will grow up to $\$ 1.7$ trillion in 2020 [1]. With the high demand for IoT devices such as smart hubs, cameras, and healthcare devices, Gartner predicts that a typical family home could contain more than 500 IoT devices by 2022 composing smart cities [2]. To deal with the data from IoT devices processed by cloud computing efficiently, fog/edge computing architecture has been considered these days.

\subsubsection{Fog/Edge Computing Architecture for Internet of Things}

Following the phenomenon of IoT generation, current cloud computing architecture is moving to fog/edge computing architecture to carry smart homes, and further smart cities. This architecture deploys edge devices to carry out the considerable amount of computation and storage locally by reducing the latency between IoT devices and servers, and improve the 

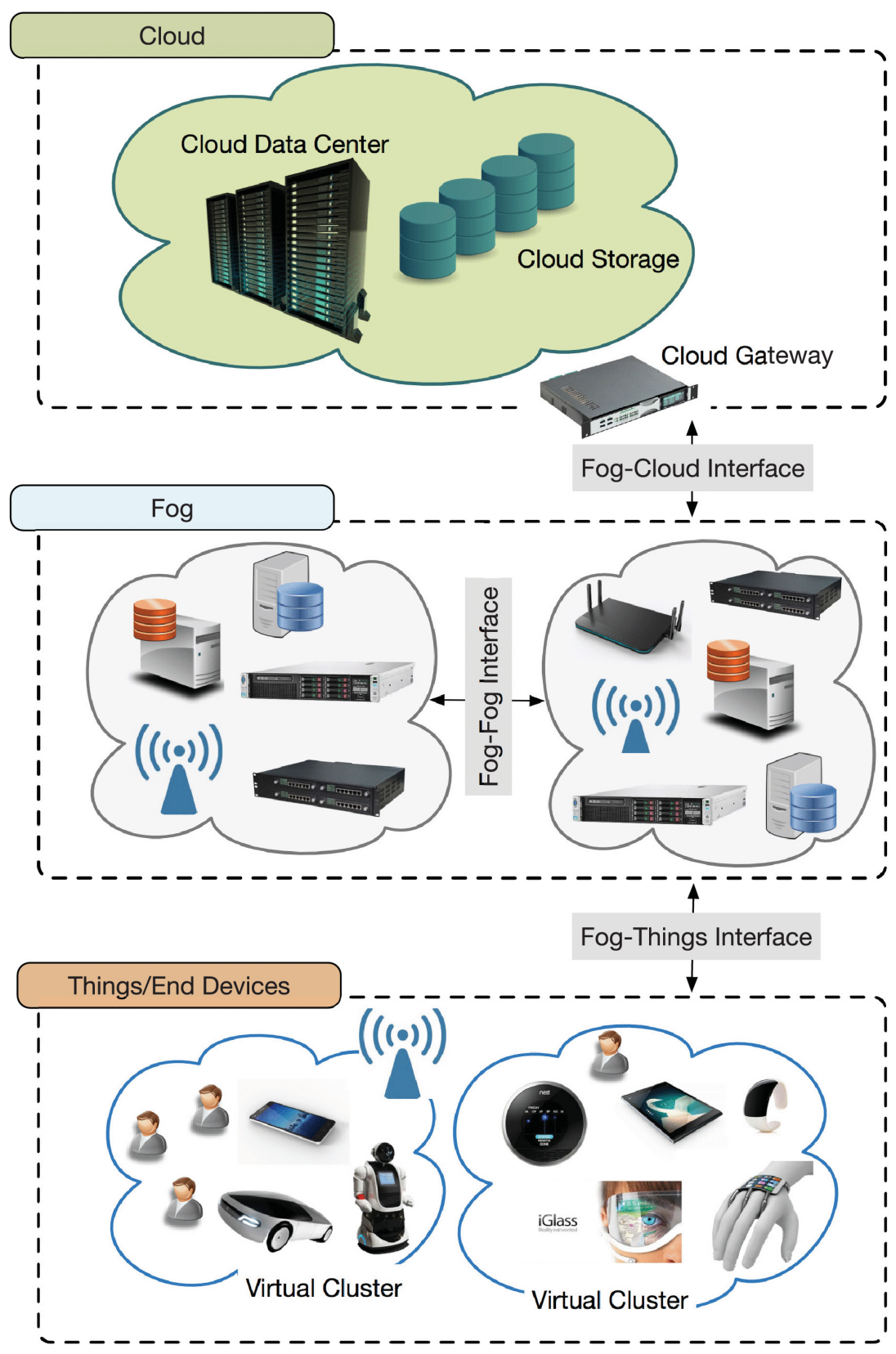

Figure 1.1: 3-tier fog computing architecture

user's experience and resilience of services in the long run [3]. Figure 1.1 describes the basic fog/edge computing architecture concisely, and it has three tiers as follows [4]:

- Tier-1 Things/End Devices : Devices of tier-1 contain IoT devices such as sensors and end user (EU)'s mobile devices including smart phones, tablets, and smart watch. These end devices are also known as Terminal Nodes (TNs). 
- Tier-2 Fog: The fog/edge nodes of tier-2 consist of the network router, switch, gateway, and Access Points (APs). In fog/edge computing architecture, these devices have the function of computation and storage.

- Tier-3 Cloud : This tier includes usual cloud servers and data centers which are capable of providing sufficient storage and computing resources.

In the middle of the architecture, the fog/edge nodes are required to bear and accommodate traffic generated by both of the cloud servers and end devices through efficient network scheduling or resource management.

\subsubsection{Active Queue Management for Internet of Things}

Although IoT devices do not have to transmit entire packets to cloud servers through the edge gateway, edge devices suffer from traffic generated by numerous IoT devices. For efficient management when it comes to queuing networks, Active Queue Management (AQM) has emerged as the intelligent network managing mechanism to selectively transmit and receive packets [5]. Unlike passive queue such as First-In-First-Out (FIFO), AQM introduces the intelligent drop of network packets to reduce network congestion by tuning AQM's parameters such as packet-drop probability adapting to the state. Widely used and recommended AQM schemes by Internet Engineering Task Force (IETF) [6] include Random Early Detection (RED) and Controlled Delay (CoDel) that will be introduced in chapter 2.

\subsubsection{Reinforcement Learning for Network Management}

In recent years, Machine Learning (ML) has become an important technology and grown up in the industries and our quality of life. And especially, Deep Learning (DL) appears to be outperforming among various ML approaches in various fields such as efficient data coding and modeling objects (unsupervised learning) as well as normal classification and prediction jobs (supervised learning) [7]. DL also has been applied to Reinforcement Learning (RL), 
which is a category of ML aiming at how software agent learns optimal actions on certain states to take the maximum cumulative reward [8]. Deep Reinforcement Learning (DRL) is DL applied RL which means that software agent's function approximator deploys Deep Neural Network (DNN) as its baseline, and it has also demonstrated greater performance on RL based applications such as playing games and motion control. Recently, the domain or application of state-of-the-art DRL schemes has been extended to networking fields such as network resource management [9].

\subsection{Motivation and Objective}

Since the state transition of network environment is discrete, this discrete nature is suitable for being represented as a finite Markov Decision Process (MDP) which is introduced in chapter 3 in detail. Because the finite MDP is the main principle of reinforcement learning, we are motivated by this point, in addition to the fact that DRL has shown remarkable performance on highly stochastic environments, and IoT infrastructure consists of numerous various types of end devices causing stochastic state transition. Our objective is to use deep learning to AQM for the first time as well as designing and implementing a fine state-aware AQM scheme on the interface of fog/edge nodes against the transilient IoT environment. Since fog/edge nodes have enough computing power, training the environment through DNN is not an issue.

\subsection{Thesis Contributions}

To enhance active queue management, we propose a deep reinforcement learning applied AQM scheme for efficient network management and study the trade-off between queuing delay and throughput, while maintaining Quality of Service (QoS) in terms of low jitter. Our system is designed based on Deep Q-Network (DQN) including main Q-network and target network trained using experience replay. It selects a packet drop or non-drop action 
at the packet departure stage depending on the current state consisting of current queue length, dequeue rate, and queuing delay. After an action is selected, a reward is calculated based on the several elements that will be clarified in chapter 3 in detail. All experiments are conducted on IoT based topology.

The main contributions of this thesis can be summarized as follows:

- Introduction of DRL applied AQM scheme for efficient network management to study the trade-off between queuing delay and throughput with maintained QoS such as jitter

- Implementation of IoT testbed which contains 21 different real IoT devices' characteristics in seven different IoT device categories

- Analysis of the experimental results, verification of our proposed scheme' s performance, and providing of design guidelines for DRL based AQM

\subsection{Thesis Organization}

The rest of this thesis is organized as follows. In Chapter 2, related works including widely used AQM schemes and ML applied AQM algorithms are described. The overview and detail design of our AQM scheme are presented in Chapter 3. Next, our IoT testbed including topology, scenarios and IoT characteristics is explained in Chapter 4. Finally, we evaluate and analyze our experiment results in Chapter 5 and we conclude the thesis with summary and future work in Chapter 6. 


\section{Chapter 2}

\section{Literature Review of Active Queue}

\section{Management}

Active Queue Management (AQM) is one of the intelligent network management methods deployed in the network interface of a router or switch [5]. AQM has parameters such as packet-drop probability tunable by both of users and queue itself adjusting to the environment. Unlike the traditional queue management methods such as drop-tail and FIFO, AQM drops packets before the queue is full to avoid the queue overflow by bursty flows as known as bufferbloat. Consequently, it causes low throughput and high latency and jitter, which is the variation in the latency or queuing delay on packet flows and that is why AQM has been developed and used widely. However, while coping with this problem, early AQM schemes had a drawback which is the parameter tuning since the performance of AQM highly depends on the base parameters setup. There have been attempts for either minimum tuning of parameters or self parameter tuning. In this chapter, we introduce the widely-used AQM and research about ML applied AQM schemes as well as the network scheduling algorithms. We also review AQM performance studies on IoT testbed. 


\subsection{Active Queue Management Schemes}

Traditional queue management cannot deal with rapidly changing queue's state such as oversized buffers which is bufferbloat. Recent IETF has expressed interest in up-to-date AQM schemes to deal with this problem [6]. Here, we briefly introduce and summarize three recognized AQM schemes: RED [10], CoDel [11], and PIE [12] recommended by IETF.

\subsubsection{Random Early Detection (RED)}

RED is one of the early classic AQM schemes for congestion avoidance, and basically it has three parameters to adapt to the state: two thresholds $t h_{\min }$ and $t h_{\max }$, and packet marking probability $p$. If the average queue size $L_{\text {avg }}$ is less than $t h_{\text {min }}$, packet is accepted to enqueue. If queue length is in between $t h_{\min }$ and $t h_{\max }$, RED calculates $p$ to mark the packet by dropping it or by modifying the packet header depending on the transport protocol. If $L_{\text {avg }}$ is greater than $t h_{\max }$, RED drops the packet rather than modifying the packet header to control $L_{\text {avg }}$. Variants of RED have been developed including Adaptive RED (ARED) and Weighted RED (WRED) for better QoS [13].

\subsubsection{Controlled Delay (CoDel)}

CoDel is packet-sojourn time based AQM, and tracks the (local) minimum queuing delay experienced by packets. The minimum packet-sojourn time can be decreased only when a packet is dequeued; and to maintain the updated minimum value, CoDel measures the value within a certain interval $T_{i n t}$, set to $100 \mathrm{~ms}$ by default. CoDel assumes a target delay $T_{\text {target }}$ and when the queuing delay exceeds $T_{\text {target }}$ during $T_{i n t}$, a packet is dropped and the next drop time is set by a control law defined as follows:

$$
t_{\text {new }}=t_{\text {curr }}+\frac{T_{\text {int }}}{\sqrt{N_{\text {drops }}}},
$$


where $t_{\text {new }}$ is new next drop time, $t_{\text {curr }}$ is current next drop time and $N_{\text {drops }}$ is the number of drops since the dropping state was entered. When the queue delay is below $T_{\text {target }}$, the controller stops dropping the packets. A distinct feature of CoDel is that it drops packets at the packet departure stage. We deal with FlowQueue-CoDel (FQ-CoDel) [14] as well, which classifies flows into one of 1024 sub-queues by hashing the 5-tuple of source and destination IP addresses, source and destination port numbers, and protocol number. Each sub-queue is implemented based on CoDel, and FQ-CoDel deploys a byte-based deficit round-robin (DRR) mechanism to serve sub-queues. FQ-CoDel has a distinct parameter quantum which is the number of bytes each queue keeps to decide to dequeue on each round of the scheduling algorithm. The default size of quantum is set to 1514 bytes, which corresponds to the Ethernet maximum transmission unit (MTU) plus the hardware header length of 14 bytes.

\subsubsection{Proportional Integral controller Enhanced (PIE)}

PIE is a burst-tolerance AQM scheme. Similar to CoDel, PIE drops the packet depending on the queuing delay, but it is more delicate with additional parameters. For every $T_{\text {update }}$ interval, PIE calculates packet-drop probability $p$ based on queuing delay, $\alpha$, and $\beta$ where $\alpha$ and $\beta$ are static configured parameters, set to 0.125 and 1.25 by default which determine the final balance between latency offset and latency jitter. When it comes to PIE, queuing delay is estimated by dequeue rate as follows:

$$
\text { cur_del }=\frac{\text { qlen }}{\text { avg_drate }}
$$

where cur_del is the current queuing delay, qlen is current queue length in bytes, and avg_drate is the average dequeue rate. To overcome rate fluctuation which is a common issue in wireless networking, PIE measures the dequeue rate periodically (refer to Appendix A for details). PIE handles bursts as well by deploying a maximum bursting allowance parameter $B_{\max }$ to allow bursts bypassing the random drop process. 
By using AQM schemes, the bufferbloat and network congestion have been alleviated comparing with using traditional passive queues, but it is still possible to improve the AQM combining different technologies such as Machine Learning (ML) dealt in the next section.

\subsection{Machine Learning Applied AQM and Network Traf- fic Scheduling Algorithms}

Machine learning (ML) is a study of data analysis that approximates an algorithm or mathematical model to improve performance of a certain task gradually. As one of ML methods, reinforcement learning $(\mathrm{RL})$ is defined as training an agent to make it select an optimal action for a certain task by giving a reward. In networking field, the agent's "certain task" can be expressed as a network scheduling or resource management. There have been several attempts for applying ML and RL techniques to not only in AQM but also in network traffic and resource control.

In [15], Bouacida et al. implemented LearnQueue AQM algorithm based on reinforcement learning for wireless networking. By manipulating a buffer size dynamically using Q-Learning in a certain interval, they update the Q-table and optimize the policy of Q-function, but they tested their algorithm with only two and three nodes deployed scenarios. Bisoy et al. of [16] suggested the AQM scheme based on a shallow neural network of one hidden layer consisting of three neurons to deal with non-linearity of networking system and to reduce queuing delay, but their work did not deal with trade-off between throughput and delay performance. In [17], Vucevic et al. proposed Reinforcement Learning-Queuing Delay Limitation (RL-QDL) AQM algorithm. The RL agents receive topology information from bandwidth broker that manages the resource handling and QoS provisioning depending on the achievement of QoS expectations in egress routers (ER). It assumes class based queuing (CBQ) by supporting three different classes: expedited forwarding (EF), assured forwarding $(\mathrm{AF})$, and best effort (BE) traffic in order to provide end-to-end QoS to users with different 
types of services.

In terms of the network scheduling algorithms, Chen et al. proposed DRL based optimized computation offloading policy by deploying a double DQN on the edge node [18]. Comparing with baseline algorithms, their approach indicated the optimal trade-off between the task delay and drops. In [19], Chinchali et al. investigated real data of cellular network traffic and introduced DRL based scheduler focusing on High Volume Flexible Time (HVFT) traffic including software and data updates to mobile IoT devices, large data transfer from IoT sensors to cloud servers, and pre-fetched ultra-high quality and bit-rate video. As a baseline of DRL model, authors used Deep Deterministic Policy Gradient (DDPG) algorithm to train actor and critic networks, and the RL agent resides at the cell tower for HVFT control. In [20], Xu et al. applied DRL to network traffic engineering by deploying actor-critic method with prioritized experience replay. Authors compared their algorithm with the widely used baseline solutions such as Shortest Path (SP), Load Balance (LB), and Network Utility Maximization (NUM), and verified their model performs better than given baseline solutions.

Although there have been meaningful works, current ML based AQM works do not consider stochastic environments such as IoT networks, or introduced network schedulers work on the task or job processing level, not packet processing level. In the next section, we review the research about traffic management for IoT applications as well.

\subsection{Traffic Management for IoT Applications}

Along with the growth of IoT markets and technologies, traffic scheduling and management for IoT applications have been flourished recently.

Kua et al. analyzed performances of several AQM schemes such as FlowQueue CoDel (FQ-CoDel) and PIE assuming the IoT enabled smart home testbed including low and high-rate IoT applications, video call traffic, bulk file transfers, and multimedia streaming 
server [21]. As the indicators of AQM schemes' efficiency, authors described throughput and round trip time (RTT). There were efforts to reduce IoT service delay for edge computation offloading policy as well [22], [23]. In [22], Zhang et al. consider G/M/1 queue as a task buffer at the edge computing server in First Come First Served (FCFS) and non-preemptive manner. The authors used Locality-First policy and probability-based policy by setting a probability parameter to make an offloading decision as the offloading policies. Yousefpour et al. used load sharing approach between edge nodes to reduce the IoT service delay as an edge offloading policy [23]. They considered the different request types from IoT devices as well as the queue length which is the task buffer for load sharing.

In [24], Zhao et al. introduced multi-tier in fog/edge layer for desired throughput-delay trade-off per user consisting of fog access node (FAN) which caches a subset of popular contents and fog control node (FCN) connected to FAN through the backhaul connections having higher computing and storage capacity than FAN. Zheng et al. investigated multiple service frequency constraints in wireless channels [25]. The authors scheduled packets arrived at links using round-robin and maximum-weight scheduling. The links are categorized into different stages indicating the priority level, and on the same stage, maximum-weight scheduling gives a priority to a link with longer queue size to be served.

In [26], Bhandari et al. applied priority queues to the cluster head to prioritize and aggregate the incoming packets. The proposed mechanism guaranteed QoS requirements in terms of latency and reliability. Al-Kashoash et al. proposed optimization-based hybrid congestion alleviation (OHCA) which combines both traffic and resource controls in the IPv6 over Low-power wireless personal area network (6LoWPAN) to utilize the network resources effectively [27]. Zhao et al. approached in a different way from above works which is laying cloudlets down on the optimal places to minimize the access delay using Software Defined Network (SDN) [28]. The control plane on SDN controllers assigns cloudlets to APs based on the average cloudlet access delay among all placement cases. 


\subsection{Summary}

In this chapter, we introduced widely used AQM schemes including RED, PIE, and CoDel and reviewed related works for network traffic and resource management based on ML techniques. Unlike the research about traffic management for IoT applications dealt in section 2.3 which is task scheduling or management, our approach deals with network flow of packets itself at the physical queue level in the network device's interface. To the best of our knowledge, this is the first work to design deep learning based AQM as well as DRL. Table 2.1 tabulates the summary of related works, and in the next chapter, we introduce the design of our system model in detail. 


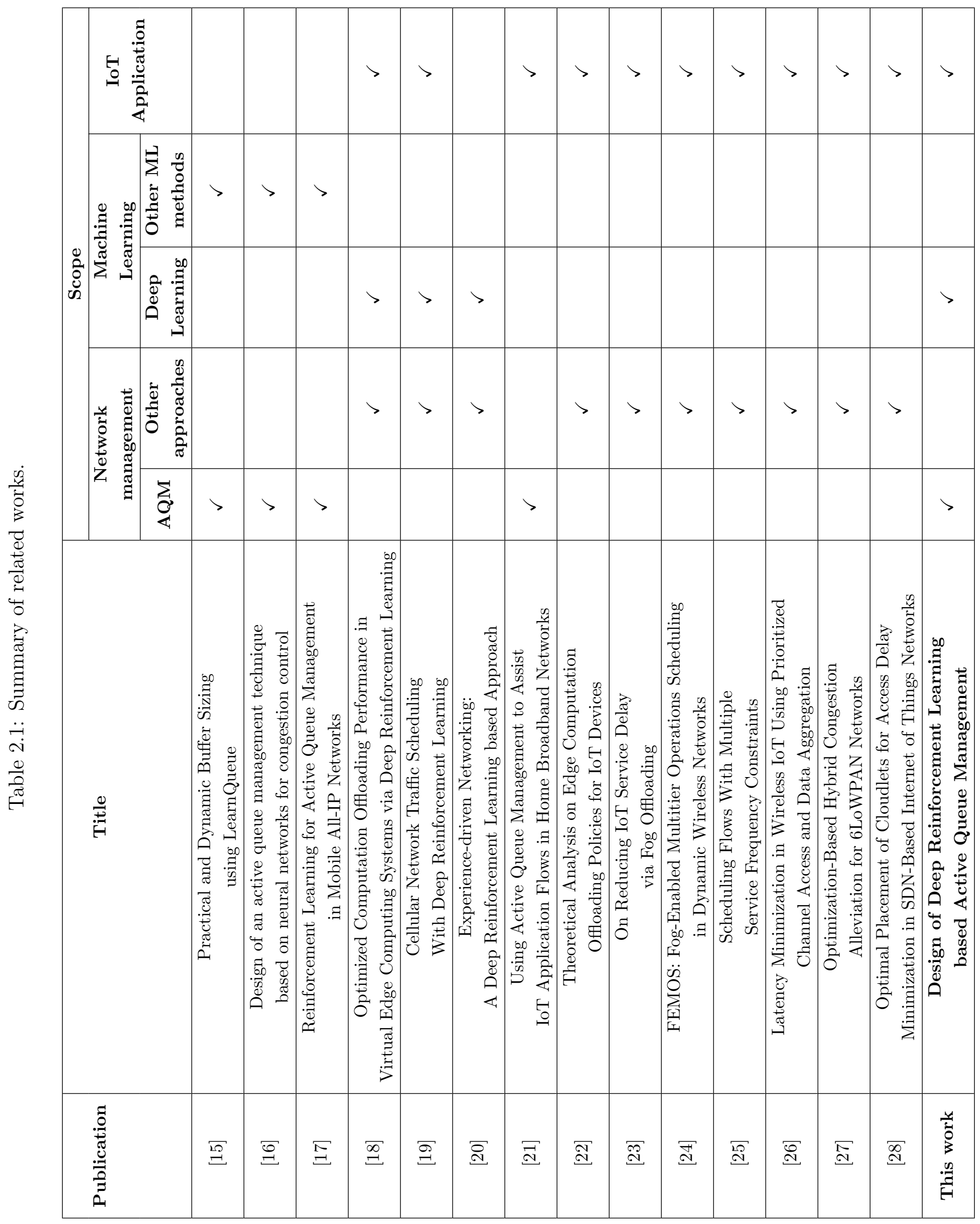




\section{Chapter 3}

\section{System Model}

In this chapter, we first give an explanation of Deep Q-Network (DQN) which is the baseline of our system. Then we describe the design of our system in terms of the state, action, and reward, and the algorithm is followed by focusing on how to give a reward to the agent in detail.

\subsection{Deep Q-Network}

In reinforcement learning, the software agent interacts with the environment $\varepsilon$ in a sequence of discrete time step $t=1,2,3, \ldots$, and generally $\varepsilon$ is stochastic. At each time step $t$, the agent selects an action $a_{t} \in A(s)$ from the set of action space on the given state $s_{t} \in S$. After taking an action $a_{t}$, the agent receives a reward $r_{t} \in R \subset \mathbb{R}$ and observes a new state $s_{t+1}$. By iterating this interaction, each observation in the sequence or trajectory is suitable for being expressed as a finite Markov Decision Process (MDP) that begins like this:

$$
s_{1}, a_{1}, r_{1}, s_{2}, a_{2}, r_{2}, s_{3}, a_{3}, r_{3}, \ldots
$$

The transition of MDP satisfies Markov property that refers to the memoryless property of a stochastic process. In the stochastic process, future states of the process only depend on the 
present state, not on the whole trajectory of events. Figure 3.1 shows the finite transition of MDP [8]. On the network queue, as packets come into the queue, the agent can observe the current queue state such as queue length and take an action whether to drop the packet or not. The queuing delay is affected by the action, and we can derive a reward as well as observe a next state. Therefore, it can be considered as MDPs, and we can apply RL to the sequence representation at each time step $t$.

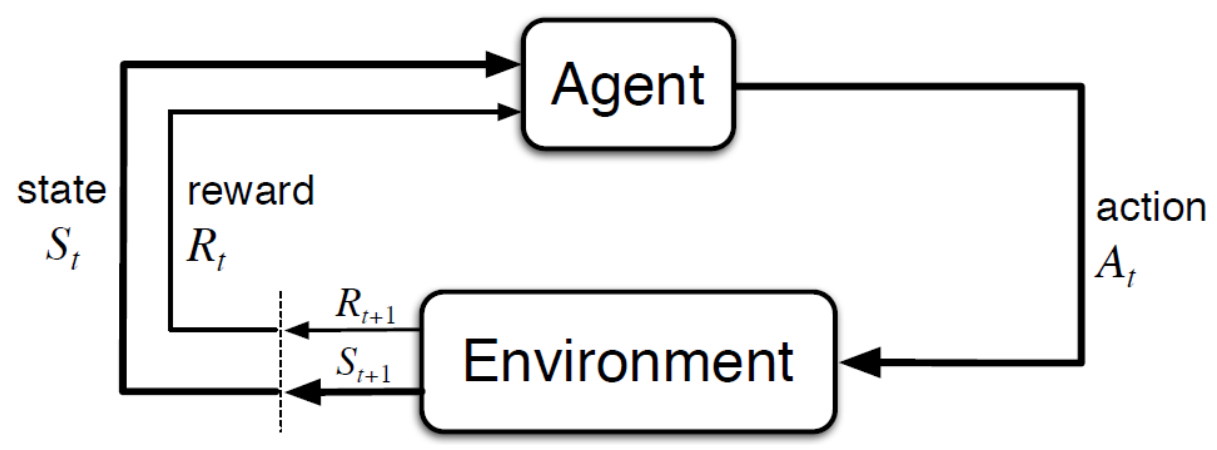

Figure 3.1: Markov Decision Process.

The two most significant features of RL are trial-and-error search and delayed reward which means that the next state's immediate reward is also considered on the current state [8]. Ultimately with these features, the agent selects an optimal action on any given state by maximizing the total reward. In order to evaluate the value of actions, Q-learning has been used as a common method of Temporal Difference (TD) learning that updates the model at the end of each time step [29]. Q-learning finds an optimal policy for expected cumulative future reward on the current state. Action-value function known as Q-function, gives a value for every action of each state, and is updated by the following equation:

$$
Q\left(s_{t}, a_{t}\right) \leftarrow(1-\alpha) * Q\left(s_{t}, a_{t}\right)+\left(r_{t}+\gamma * \max _{a^{\prime}} Q\left(s_{t+1}, a^{\prime}\right)\right)
$$

where $r_{t}$ is a reward after taking an action $a_{t}$ on state $s_{t}, a^{\prime}$ is an expected action to maximize the Q-value, $\alpha$ is learning rate, and $\gamma$ is discount factor for future reward. Since we design discrete actions of packet drop/non-drop actions, our policy $\pi=p(a \mid s)$ is deterministic that 
maps each state $s$ to an action $a$. The goal of the agent is to select an action to maximize the cumulative future reward through Q-function, so the optimal Q-function can be expressed as follows:

$$
Q^{*}(s, a)=\max _{\pi} \mathbb{E}_{s_{t} \sim \varepsilon}\left[r_{t}+\gamma r_{t+1}+\gamma^{2} r_{t+2}+\ldots \mid s_{t}=s, a_{t}=a, \pi\right]
$$

which is the maximum expected cumulative reward acquired by the policy $\pi$ after taking an action $a$ on the state $s$. Since we focus on deep neural network (DNN) as a function approximator, it outputs the predicted Q-value $Q(s, a \mid \theta)$ where $\theta$ s are weights of neurons of DNN. In order to minimize the difference between predicted Q-value and optimal Q-value, loss is defined as:

$$
L(\theta)=\min _{\theta} \sum_{t=1}^{T}\left[\left(Q\left(s_{t}, a_{t} \mid \theta\right)-\left(r_{t}+\gamma * \max _{a^{\prime}} Q\left(s_{t+1}, a^{\prime} \mid \theta\right)\right)\right)^{2}\right]
$$

However, when reinforcement learning uses a neural network as a function approximator, it has a disadvantage that is unstable or diverges to train Q-function. One reason of the instability is that the neural network receives highly correlated data as sequential inputs in the observation, so it causes over-fitting or falling into local minimum. Another reason of divergence is due to non-stationary target which means that both of the predicted Q-value and optimal Q-value depend on weights $\theta$ so when the neural network is trained, target value is also changed and it is difficult to converge. To overcome these issues, DQN has been developed, which is the baseline model of our system [30]. There are two main features of DQN: experience replay and network separation.

In order to reduce correlation between observations, the concept of experience replay is used. Instead of training the neural network in order of sequence, we store the agent's experiences $e_{t}=\left(s_{t}, a_{t}, r_{t}, s_{t+1}\right)$ at each time step $t$ into the replay memory $D_{t}=\left\{e_{1}, e_{2}, \ldots, e_{t}\right\}$. To update the neural network, mini-batches of experiences $\left(s, a, r, s^{\prime}\right) \sim U(D)$ are selected randomly in uniform distribution from the replay memory. The way of training the neural 
network at iteration $i$ uses a similar loss function to the equation (3.3):

$$
L_{i}\left(\theta_{i}\right)=\mathbb{E}_{\left(s, a, r, s^{\prime}\right) \sim U(D)}\left[\left(Q\left(s, a \mid \theta_{i}\right)-\left(r+\gamma * \max _{a^{\prime}} \hat{Q}\left(s^{\prime}, a^{\prime} \mid \theta_{i}^{-}\right)\right)\right)^{2}\right]
$$

By deploying two networks (main Q-network $Q$ and target network $\hat{Q}$ ), we can set the stationary target. The target network parameters $\theta_{i}^{-}$are updated by Q-Network parameters $\theta_{i}$ periodically, not in every step. With these features, DQN has shown outstanding performances comparing with all previous Q-learning based algorithms as will be seen in chapter 5 .

\subsection{DQN based AQM System Design}

In this section, we introduce the design of state, action, and reward function for our DQN based AQM algorithm.

\subsubsection{Process of Selecting Action}

With respect to the state of RL, we consider three elements: current queue length in packets $L$, dequeue rate $R_{d e q}$, and queuing delay $d$ and we use PIE's dequeue rate calculation method [12]. At each time step $t$, state $s_{t}$ is defined as $s_{t}=\left\{L_{t}, R_{d e q, t}, d_{t}\right\}$ which is an input of multilayer perceptron (MLP) consisting of two hidden layers of 64 neurons for each layer. For selecting an action, main Q-network is used and it returns two probabilities as an output (drop/non-drop probability). Following the higher probability, it decides a packet drop/nondrop action $a_{t}$, and the action occurs on the packet departure stage of queue as if CoDel's dropping action [11]. In order to find a better action on a certain state, we use explore/exploit strategy which means that the agent takes an action based on its own selection (exploit), or sometimes takes a random action uniformly based on a certain probability (explore). For the explore/exploit strategy, we use $\epsilon$-greedy algorithm starting from a highly random action probability. At the first episode of the network simulation, the exploring probability is set 
to $90 \%$ based on the round of episode and it dwindles down to $0 \%$ through the episodes. The agent takes an action in periodic interval $T_{\text {int }}$ only when there is queuing delay in the queue. Figure 3.2 describes the process of selecting an action.

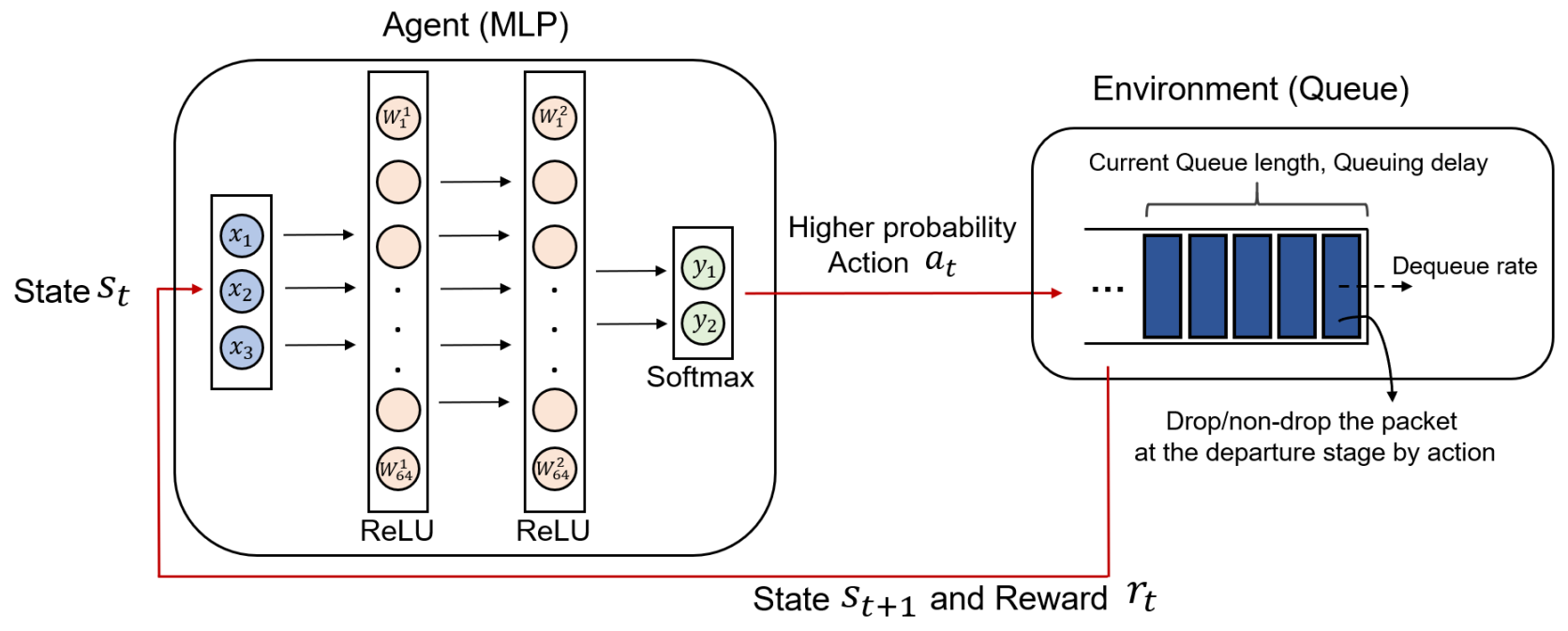

Figure 3.2: Process of selecting an action.

\subsubsection{Reward Engineering}

After taking an action, the RL agent waits for next state $s_{t+1}$ during the interval $T_{\text {int }}$. The selected action is evaluated by a reward function. The most important point of designing the reward function is to optimize the trade-off between queuing delay and drop-rate as well as to avoid infinite packet drop state or non-drop state. We refer learnQueue's reward function as a baseline [15]. There are two main components for a reward: delay_reward and enq_reward for queuing delay and packet drop-rate respectively. The delay_reward is defined as:

$$
\text { delay_reward }=\delta *(\text { delay_ref }- \text { curr_delay })
$$

where delay_ref is the desired queuing delay, curr_delay is the current queuing delay defined in the equation 2.2, and $\delta$ is a scaling factor between delay_reward and enq_reward. The 
enq_reward is defined as:

$$
\text { enq_reward }=(1-\delta) *(\text { min_delay }- \text { delay_ref }) * e n q \_r a t e,
$$

where enq_rate is enqueue rate during $T_{\text {int }}$, and it is calculated as:

$$
\text { enq_rate }=\frac{N_{e n q}}{N_{e n q}+N_{\text {drops }}}
$$

where $N_{\text {enq }}$ is the number of enqueued packets and $N_{\text {drops }}$ is the number of dropped packets. When $N_{e n q}$ is 0, enq_rate is set to 0 . Depending on the scaling factor $\delta$, we can balance the importance between the queuing delay and packet drop-rate by the agent. Unlike learnQueue's reward function that defines max_delay which is the maximum time required to drain the queue depending on the $\mathrm{AP}^{\prime}$ s physical data rate, we define min_delay which is delay when the link between the edge device and cloud gateway is fully utilized as follows:

$$
\text { min_delay }=\frac{L_{\text {byte }}}{R_{\text {phy }}}
$$

where $L_{\text {byte }}$ is the current queue length of the edge device in bytes, and $R_{\text {phy }}$ is the physical bandwidth (data rate) of peer-to-peer (P2P) link connected to the edge device. Finally, the reward is calculated as the sum of both components:

$$
\text { reward }=\operatorname{clip}(\text { delay_reward }+ \text { enq_reward },-1,+1) .
$$

To avoid the divergence of a reward and regularize it, we clip the reward to the constant minimum and maximum values corresponding to -1 and +1 . 


\subsubsection{Training Process}

Since we use DQN as our model, the agent stores an experience $e_{t}=\left(s_{t}, a_{t}, r_{t}, s_{t+1}\right)$ in tuple format to the replay memory at each time step $t$. Once the number of experiences in replay memory is over mini-batch size, the agent randomly picks samples of the experiences from the memory in uniform manner. Following equation (3.4), the agent minimizes the temporal difference error (TD-error) using mean square error (MSE) loss function. We apply the rectified linear unit (ReLU) activation function defined as $f(x)=\max (0, x)$ at each hidden layer, and softmax function at the output layer to convert outputs into action probabilities. At the first episode, we initialize the weights of MLPs using Xavier initializer which is assigning the weights of the layers from a Gaussian distribution [31], and optimize the loss using Adam optimizer [32] to train the model. Figure 3.3 shows the flowchart of DQN based AQM training process. In the flowchart, $t_{\text {curr }}$ is current time step $t$, and $C$ is target update step to update the target network periodically. Algorithm 1 describes the pseudocode of DQN based AQM training process. We follow the principle of DQN introduced in [30], but since there is no concept of "game over" in network simulation, the discounted future reward is always added to the immediate reward. To avoid infinite packet drop/non-drop action on certain states, we control the reward function. After giving the reward, we initialize the dequeue rate depending on the queuing delay following the PIE's dequeue rate calculation scheme [12]. In the pseudocode, we assume that in time step $t$, if the selected action is dropping the packet, $a_{t}=1$, otherwise, $a_{t}=0$. The interval of each time step is $T_{\text {int }}$.

\subsection{Summary}

In this chapter, we presented our system model in terms of the RL state and action setup, and reward function. Also we introduced which ML techniques we use to our MLPs including the activation functions, loss optimizer, and MLPs' weight initializer. In the next chapter, we introduce how we set up the IoT testbed, assumptions for the experiment, and parameters 
setup for the topology and AQM schemes.

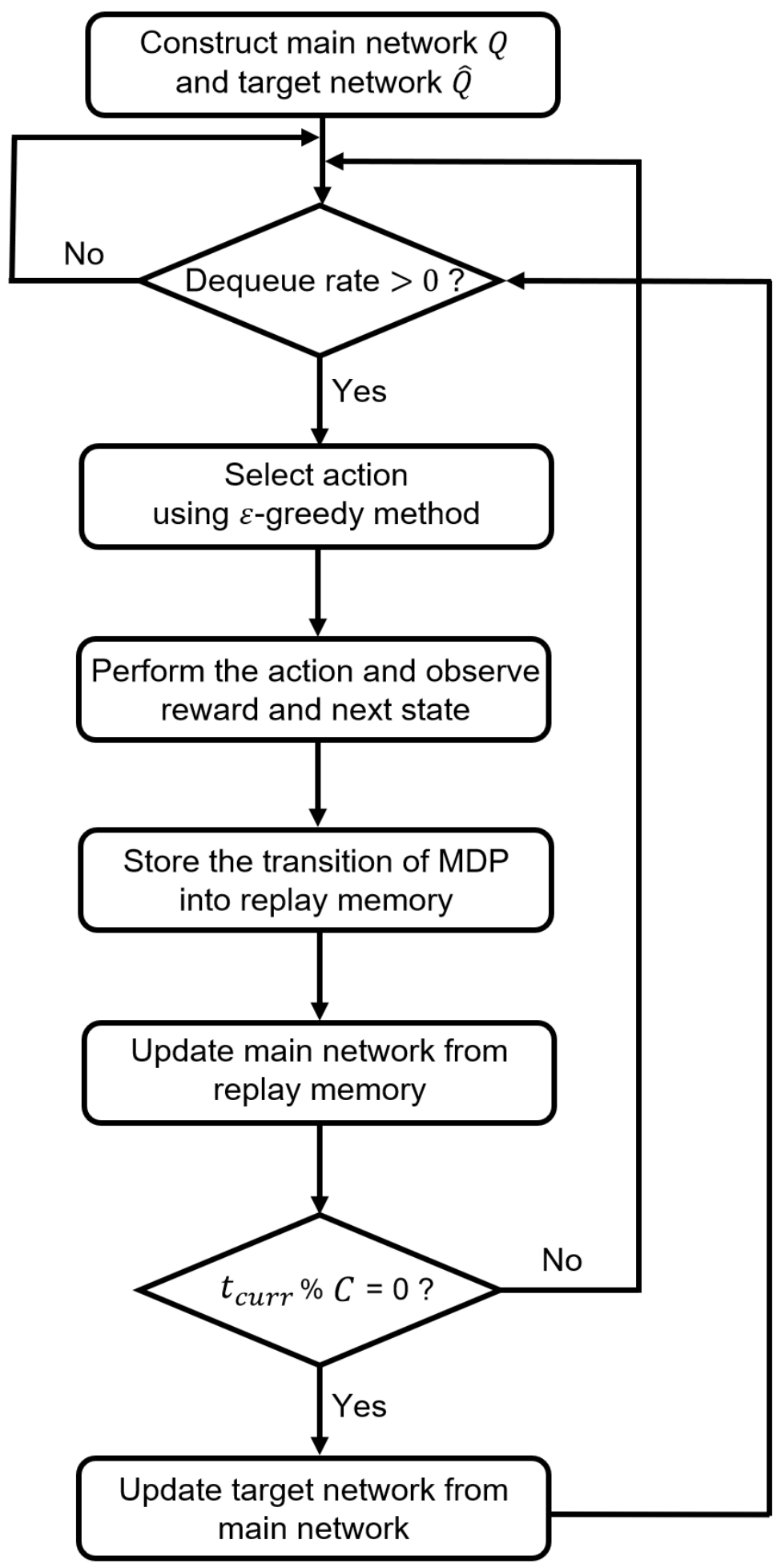

Figure 3.3: Flowchart of DQN based AQM training 


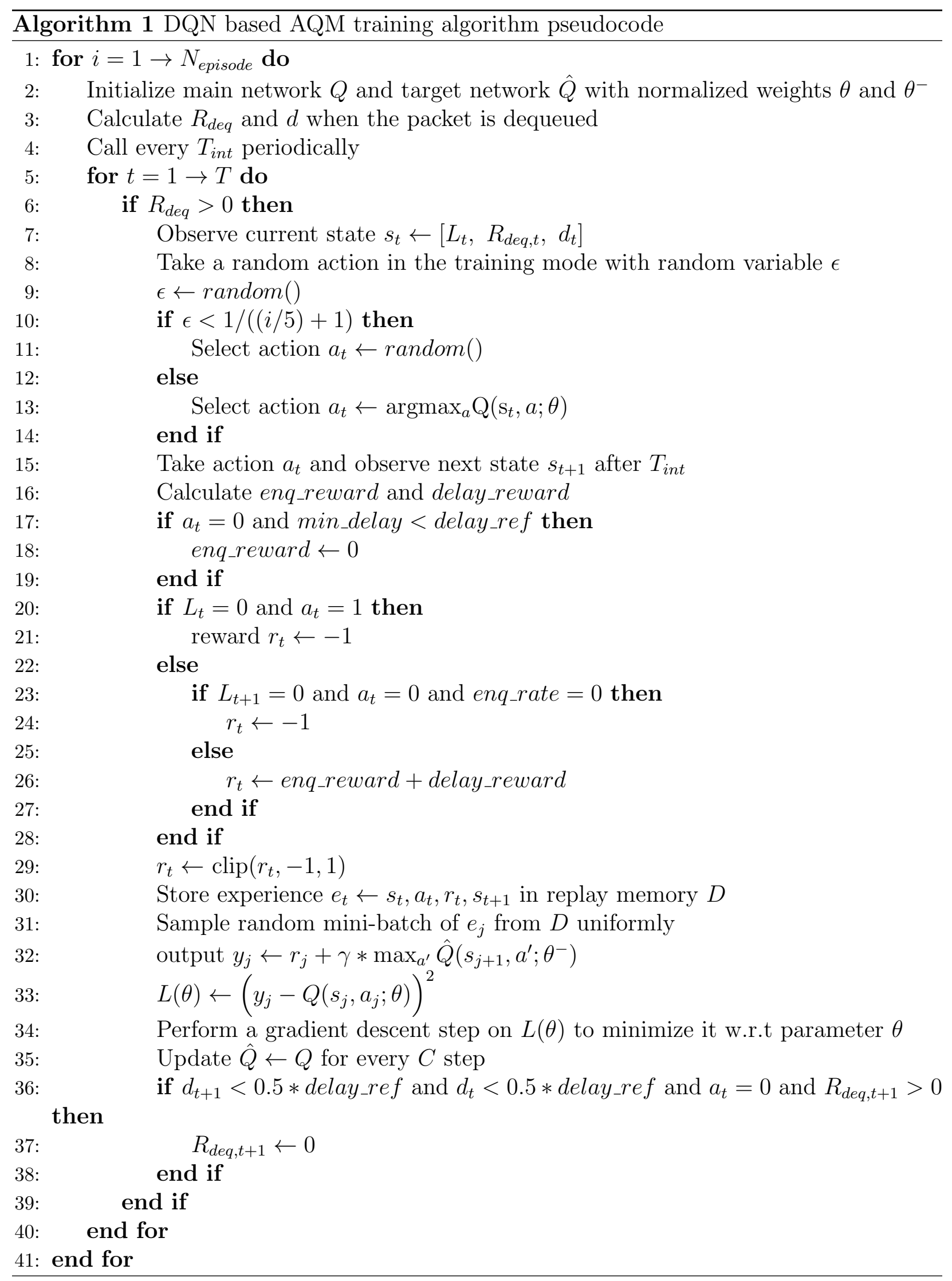




\section{Chapter 4}

\section{Experiment Setup}

In this chapter, we briefly explain the characteristics of IoT, and deal with our testbed, assumptions for the experiment, and how we set up the parameters.

\subsection{Characteristics of IoT devices}

In order to understand real behaviours of IoT devices, we survey the materials of analysis on smart home/campus network traffic. Authors in [33] measured the IoT network traffic for over three weeks, and clustered their characteristics applying K-means clustering algorithm with over $95 \%$ for IoT devices identification. After classification, they investigated the patterns of IoT as well. Their analysis shows that IoT devices remain idle for most of the time in general which means that no packet is exchanged during this interval. Also considering only IoT devices, daily average load is $66 \mathrm{kbps}$ which is higly low rate. Another observation is that most of the traffic by IoT is based on TCP, rather than UDP, and more than $50 \%$ of TCP port is 443 which is Hyper Text Transfer Protocol Secure (HTTPS)

protocol. In [34], authors investigated traffic from smart home consisting of sensors, hubs, plugs, and electronics for 22 days, and it shows similar pattern with [33]'s result. 


\subsection{IoT Testbed}

In order to construct the IoT testbed as close as possible to the real IoT infrastructure, we use real IoT characteristics clustered by [33]. From the clustered attributes, we use five characteristics to implement IoT testbed: Sleep time, Active time, Average packet size, Mean data rate, and Peak/Mean rate. Table 4.1 shows clustering of real IoT attributes, and Table 4.2 describes the measure of attribute clusters. In the topology, all IoT devices are connected to the edge device wirelessly, and the edge device is connected to the cloud gateway on $\mathrm{P} 2 \mathrm{P}$ link. The gateway transmits the received packets to destination cloud servers through carrier-sense multiple access (CSMA) channel. For the camera category of IoT attributes, we set User Datagram Protocol (UDP) assuming real-time monitoring traffic, and for other categories, we set Transmission Control Protocol (TCP) assuming widely used HTTPS protocol for IoT to generate traffic. In terms of TCP congestion control algorithm, TCP CUBIC [35] and Proportional Rate Reduction (PRR) recovery algorithm [36] are deployed which are implemented and used by default in Linux kernels. Figure 4.1 depicts our topology design.

\subsection{Assumptions and Parameter Setting}

Due to the traffic characteristics of IoT such as periodic packet transmission and small size of packets, the queuing delay or packet loss rarely happens in the normal operation of mean data rate. We assume that IoT devices burst with sending packets in peak transmission rate to occur queuing delay and packet loss, and the distance from each IoT device to the edge device is equal and constant. We regulate the round-trip time (RTT) by controlling the baseline delay of P2P link and set the low link capacity between the edge device and cloud gateway to measure the performance of AQM schemes from various perspectives assuming the narrow bandwidth for IoT devices.

All tested AQM schemes are deployed on the interface of edge node connected to the 

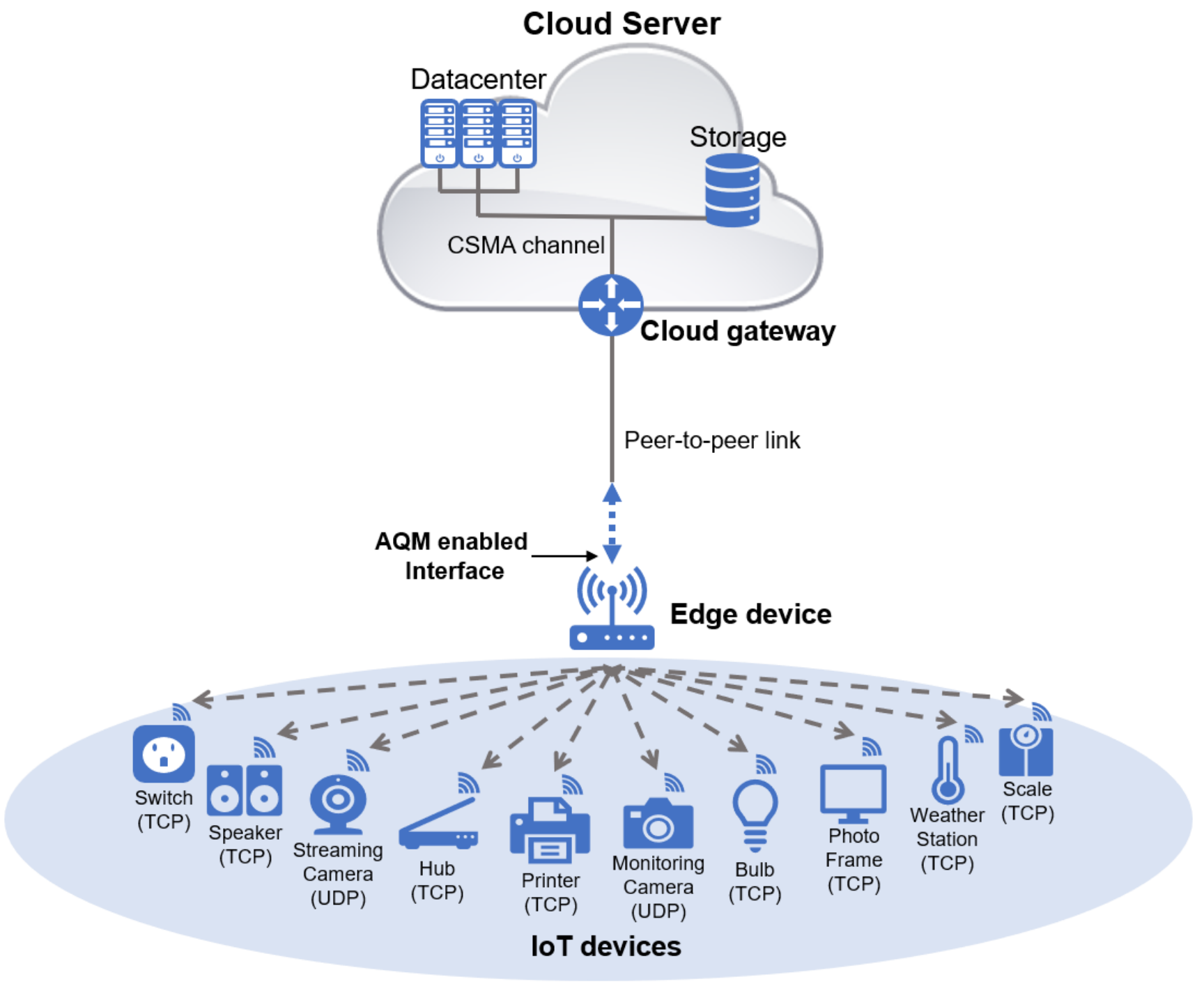

Figure 4.1: Overview of the IoT testbed

cloud gateway since the P2P link between them is set as a bottleneck link. Priority-FIFOfast queuing discipline (P-FIFO), which has three priority bands based on the packet priority and is the default priority queue on Linux systems, is deployed on all other interfaces. To conduct the experiments, we set different RTTs, P2P link capacity, reward scaling factor $\delta$, and the number of IoT devices connected to the edge device. The basic number of IoT devices that will be tested on the experiment is 21 , which is the number of clustered real IoT devices. Also we test on three times the basic number of IoT devices which is 63, to observe the performance of each AQM on more extreme congestion. The testbed is implemented 
on $n s-3$ network simulator [37], and we apply tiny-dnn which is a $\mathrm{C}++$ based open source deep learning framework to $n s-3$ [38]. Tables 4.3 and 4.4 describe the environmental setup parameters and comparison AQM setup parameters respectively. Mainly we set default parameters for the AQM schemes, but we only adjust PIE's mean packet size and dequeue threshold parameters adapting to the IoT testbed. In Table 4.4, maxSize is maximum queue size, meanPktSize is mean packet size set by user to determine each AQM's behaviours, and delay_ref is the desired queuing delay in common. In RED, the parameter LInterm is the maximum probability of dropping a packet. In PIE, dq_threshold is the dequeue threshold that indicates if there are sufficient data in queue to calculate dequeue rate. Because PIE's authors recommend dq_threshold to be set 10 times of higher size than meanPktSize, we set dq_threshold to 3500 [12]. In CoDel, minByte is the minimum bytes in queue to allow a packet drop. In FQ-CoDel, the variable Flows is the number of sub-queues for classifying flows. Table 4.5 indicates the DQN based AQM algorithm setup parameters.

Table 4.1: Clustering of IoT attributes.

\begin{tabular}{|c|c|c|c|c|c|c|c|c|c|c|c|c|c|c|c|c|c|c|c|c|c|}
\hline $\begin{array}{l}\text { IoT Device } \\
\text { Category }\end{array}$ & \multicolumn{2}{|c|}{ Hubs } & \multicolumn{6}{|c|}{ Cameras } & \multicolumn{4}{|c|}{ Switches \& triggers } & \multicolumn{2}{|c|}{$\begin{array}{l}\text { Air quality } \\
\text { sensors }\end{array}$} & \multicolumn{3}{|c|}{$\begin{array}{c}\text { Healthcare } \\
\text { devices }\end{array}$} & \multirow{2}{*}{ 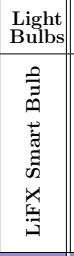 } & \multicolumn{3}{|c|}{ Electronics } \\
\hline IoT Device & 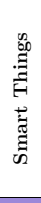 & 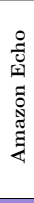 & 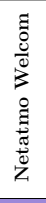 & 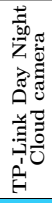 & 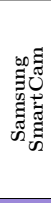 & 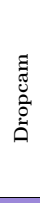 & 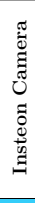 & 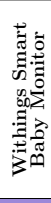 & 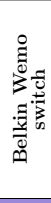 & 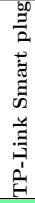 & : & 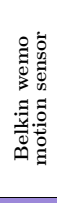 & 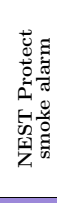 & 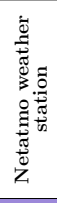 & 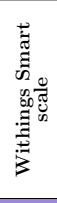 & 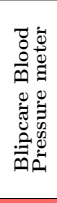 & 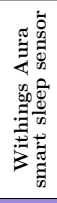 & & 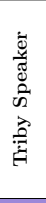 & 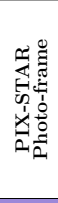 & 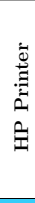 \\
\hline \begin{tabular}{|l|} 
Sleep time \\
\end{tabular} & 1 & 1 & 1 & 2 & 1 & 1 & 2 & 1 & 1 & 3 & 2 & 1 & 1 & 1 & 1 & 5 & 1 & 1 & 1 & 1 & 2 \\
\hline Active time & 1 & 1 & 1 & 1 & 1 & 1 & 1 & 1 & 1 & 1 & 2 & 1 & 1 & 1 & 2 & 3 & 1 & 1 & 1 & 1 & 1 \\
\hline Avg. Pkt Size & 1 & 2 & 4 & 1 & 4 & 2 & 2 & 1 & 4 & 2 & 1 & 3 & 3 & 3 & 3 & 2 & 2 & 1 & 2 & 3 & 1 \\
\hline Mean data rate & 1 & 1 & 2 & 1 & 2 & 1 & 1 & 1 & 3 & 1 & 1 & 3 & 2 & 1 & 1 & 1 & 1 & 1 & 1 & 1 & 1 \\
\hline Peak / Mean rate & 1 & 1 & 2 & 1 & 1 & 2 & 1 & 1 & 1 & 1 & 1 & 1 & 1 & 1 & 1 & 1 & 2 & 1 & 2 & 1 & 1 \\
\hline
\end{tabular}

\subsection{Summary}

In this chapter, we introduced our IoT testbed by explaining which inherent characteristics of IoT devices we bring in for the experiments, and parameters we set for the network simulation. In the following chapter, we analyze the empirical results of the simulation, and verify the outstanding performance of our DQN based AQM scheme. 
Table 4.2: Measure of attribute clusters.

\begin{tabular}{|c|r|r|r|r|r|}
\hline Clusters & $\begin{array}{c}\text { Sleep time } \\
\text { (Sec) }\end{array}$ & $\begin{array}{c}\text { Active time } \\
\text { (Sec) }\end{array}$ & $\begin{array}{c}\text { Avg. Pkt } \\
\text { Size (B) }\end{array}$ & $\begin{array}{c}\text { Mean rate } \\
\text { (Bps) }\end{array}$ & $\begin{array}{c}\text { Peak/ } \\
\text { Mean rate }\end{array}$ \\
\hline $\mathbf{1}$ & 4 & 1 & 94 & 462 & 11 \\
\hline $\mathbf{2}$ & 66 & 2 & 144 & 2,461 & 66 \\
\hline $\mathbf{3}$ & 241 & 8 & 234 & 11,388 & 229 \\
\hline $\mathbf{4}$ & 7,985 & 25 & 327 & 42,493 & 474 \\
\hline $\mathbf{5}$ & 24,832 & 34 & 699 & 516,540 & 1,253 \\
\hline
\end{tabular}

Table 4.3: Environmental setup parameters.

\begin{tabular}{|c|c|}
\hline Parameter & Value \\
\hline Number of servers in cloud & 2 \\
\hline CSMA channel data rate in cloud & $100 \mathrm{Mbps}$ \\
\hline P2P link data rate & $0.5,1 \mathrm{Mbps}$ \\
\hline Base RTT on P2P link & $0,10,20,50,100 \mathrm{~ms}$ \\
\hline Number of IoT devices & 21,63 \\
\hline Wi-Fi standard & IEEE $802.11 \mathrm{n} 5 \mathrm{GHz}$ \\
\hline Distance between IoT devices and edge device & $30 \mathrm{~m}$ \\
\hline Simulation time & $100 \mathrm{~s}$ \\
\hline
\end{tabular}


Table 4.4: Comparison AQM setup parameters.

\begin{tabular}{|c|c|}
\hline $\mathrm{AQM}$ & Parameters \\
\hline P-FIFO & $\max S i z e=1000$ \\
\hline RED & $\begin{array}{c}\text { maxSize }=25 p \\
\text { meanPktSize }=500 \\
t h_{\min }=5 \\
\text { th } h_{\max }=25 \\
\text { LInterm }=50\end{array}$ \\
\hline PIE & $\begin{array}{c}\text { maxSize }=25 p \\
\text { meanPktSize }=350 \\
\text { dq_threshold }=3500 \\
\alpha=0.125 \\
\beta=1.25 \\
T_{\text {update }}=0.03 \\
\text { delay_ref }=20 \mathrm{~ms} \\
B_{\text {max }}=0.1\end{array}$ \\
\hline CoDel & $\begin{array}{c}\text { maxSize }=50000 \text { bytes } \\
\text { delay_ref }=5 \mathrm{~ms} \\
T_{\text {int }}=100 \mathrm{~ms} \\
\text { minByte }=50\end{array}$ \\
\hline FQ-CoDel & $\begin{array}{c}\max \text { Size }=10240 p \\
\text { Flows }=1024\end{array}$ \\
\hline
\end{tabular}

Table 4.5: DQN based AQM algorithm setup parameters.

\begin{tabular}{|c|c|}
\hline Parameter & Value \\
\hline Number of hidden layers & 2 \\
\hline Number of neurons in each hidden layer & 64 \\
\hline Learning rate $\alpha$ & 0.01 \\
\hline Mini-bacth size & 32 \\
\hline Reward discount factor $\gamma$ & 0.99 \\
\hline Periodic target network update step $C$ & 5 \\
\hline Maximum queue size & 50 packets \\
\hline Action selection interval $T_{\text {int }}$ & $1 \mathrm{~ms}$ \\
\hline Desired queuing delay & $30 \mathrm{~ms}$ \\
\hline Reward scaling factor $\delta$ & $0.4,0.5,0.6$ \\
\hline
\end{tabular}




\section{Chapter 5}

\section{Results and Analysis}

In this chapter, we analyze and discuss the results of experiments described in chapter 4. We compare our proposed AQM algorithm with P-FIFO and various AQM schemes including RED, PIE, CoDel, and FQ-CoDel on different environments by adjusting RTT and bandwidth of P2P link between the edge node and cloud gateway.

\subsection{Comparison on Queuing Delay and Occupancy}

In this section, we study the queuing delay and queue occupancy of each AQM scheme. Figure 5.1 shows the empirical cumulative distribution function (CDF) of delay which is induced RTT that the IoT devices' flows suffer during the simulation time, and comparison of each AQM scheme. We can observe that P-FIFO mostly experiences high delay of over one second from both cases of the 21 and 63 IoT devices scenarios because it just classifies packets, but does not work actively on the fluctuating congestion. In both cases, DQN based AQM performs better than other AQM schemes. RED and PIE adapt their queue for

delay smaller or slightly larger than 500ms. Comparing with them, DQN based AQM has higher probability of queuing delay under $500 \mathrm{~ms}$ than other AQM schemes' probabilities as well as maintaining less than $500 \mathrm{~ms}$ in total. If queuing delay of $500 \mathrm{~ms}$ is required under 1Mbps bandwidth, P-FIFO, RED, PIE, CoDel, FQ-CoDel and DQN based AQM provide 
such guarantee with 4.11\%, 99.83\%, 99.99\%, 73.87\%, 79.48\%, and 100\% respectively for 21 IoT devices, and 1.09\%, 100\%, 100\%, 47.27\%, 48.79\%, and 100\% respectively for 63 IoT devices. Alternatively, in $99 \%$ of probability, maximum delays increased for 21 IoT devices are $3332 \mathrm{~ms}$, 472ms, 478ms, 696ms, 2301ms, and 388ms respectively for P-FIFO, RED, PIE, CoDel, FQ-CoDel, and DQN based AQM schemes.

Figure 5.2 depicts the queue occupancy of each AQM schemes during the simulation time of 100s. We can observe that DQN based AQM does not exceed its maximum queue size of 50 packets as an optimal behaviour for maximum future cumulative reward.

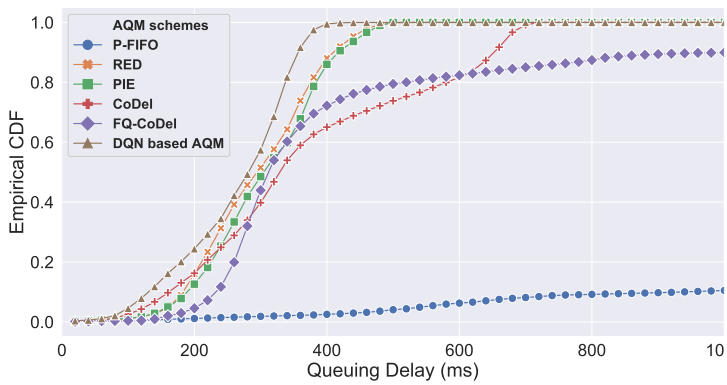

(a) 21 IoT devices

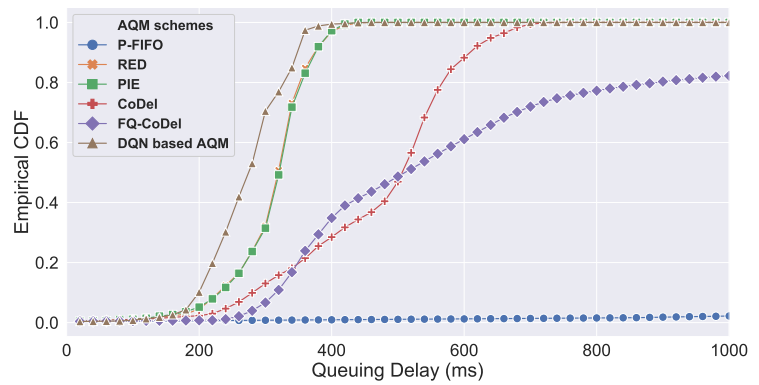

(b) 63 IoT devices

Figure 5.1: Empirical CDF of queuing delay for flows of IoT devices; P2P link data rate = 1Mbps; Baseline RTT $=0 \mathrm{~ms}$; Reward scaling factor $\delta=0.5$

\subsection{Comparison on Different Bandwidth}

Here, we analyze the performance of AQM schemes on the topology consisting of 21 IoT devices using $1 \mathrm{Mbps}$ and $0.5 \mathrm{Mbps} \mathrm{P} 2 \mathrm{P}$ link capacity between the edge device and cloud gateway. Figure 5.3 is a boxplot of the throughput for each flow. Since the IoT devices deployed in our testbed vary and transmit packets stochastically, a large number of outlier points are observed comparing with related works and the boxplot could be inconspicuous, but this graphical measurement is relatively efficient. In Figure 5.3, medians of throughput for each AQM schemes are relatively similar. FQ-CoDel shows higher median throughput on $0.5 \mathrm{Mbps}$ bandwidth, but also undergoes the second highest delay depicted in Figure 5.4 


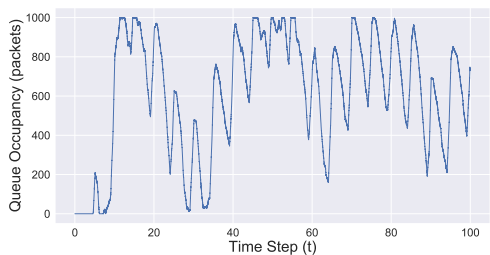

(a) P-FIFO

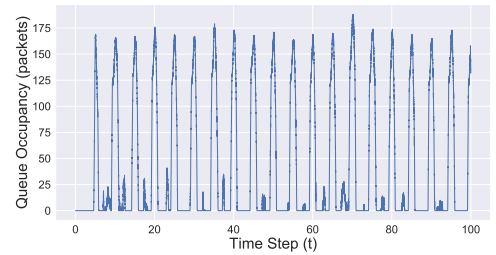

(d) CoDel

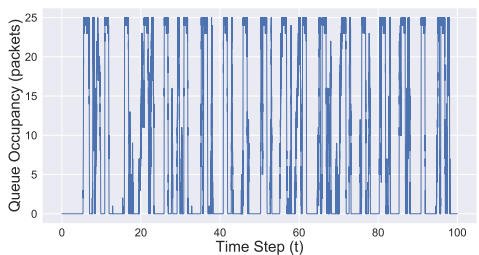

(b) RED

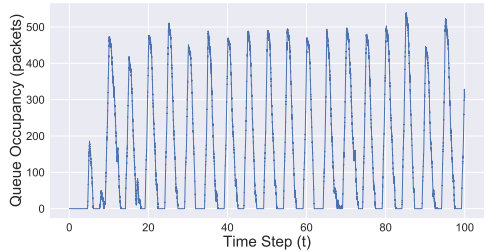

(e) FQ-CoDel

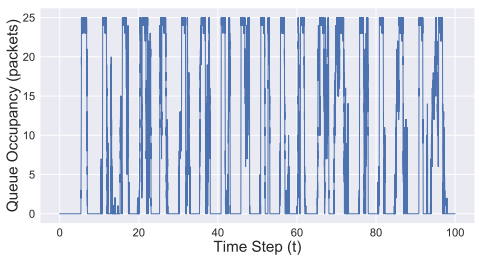

(c) PIE

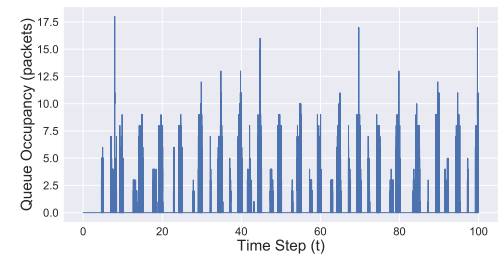

(f) DQN based AQM

Figure 5.2: Comparison of queue occupancy of AQM schemes; P2P link data rate $=1 \mathrm{Mbps}$; Baseline RTT $=0 \mathrm{~ms}$; Number of IoT devices $=21$; Reward scaling factor $\delta=0.5$

although the shape of all other delay boxplots AQM schemes' delay is too flat due to the P-FIFO. DQN based AQM shows the lowest median delay maintaining usual throughput comparing with other AQM schemes on 1Mbps bandwidth, and tenable performance of both throughput and delay on $0.5 \mathrm{Mbps}$ bandwidth.

\subsection{Comparison on Different Baseline RTT}

In this section, we compare the AQM schemes on different baseline RTTs: 0, 10, 20, 50, 100ms. Also, we set 63 IoT devices consisting of three duplicated devices from 21 distinct devices. Figure 5.5 shows each of boxplot of throughput for different baseline RTTs. We can observe that the inter-quartile range of boxplot is decreasing as the baseline RTTs become larger. The intriguing point is that the median of FQ-CoDel maintains higher throughput on every tested case. However, as a trade-off, FQ-CoDel shows high delay on every case as shown in Figure 5.6. We also investigate the maximum delay time and most frequent delay time that each flow suffers to clarify the degree of delay in Figures 5.7 and 5.8. We can see that DQN based AQM experiences low delays w.r.t. frequency and maximum values, 


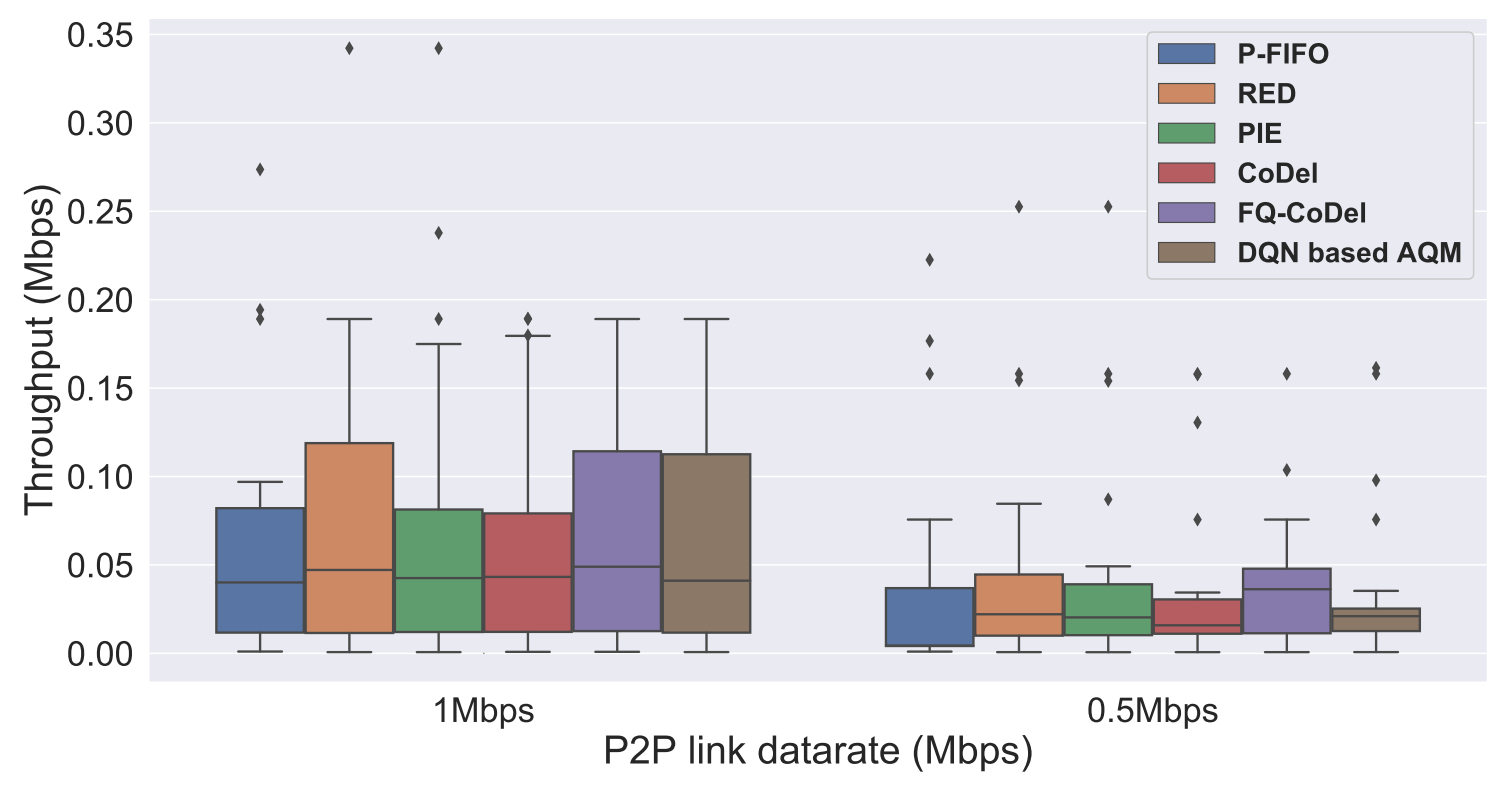

Figure 5.3: Throughput per flows of 21 IoT devices; Baseline RTT = 0ms; Reward scaling factor $\delta=0.5$

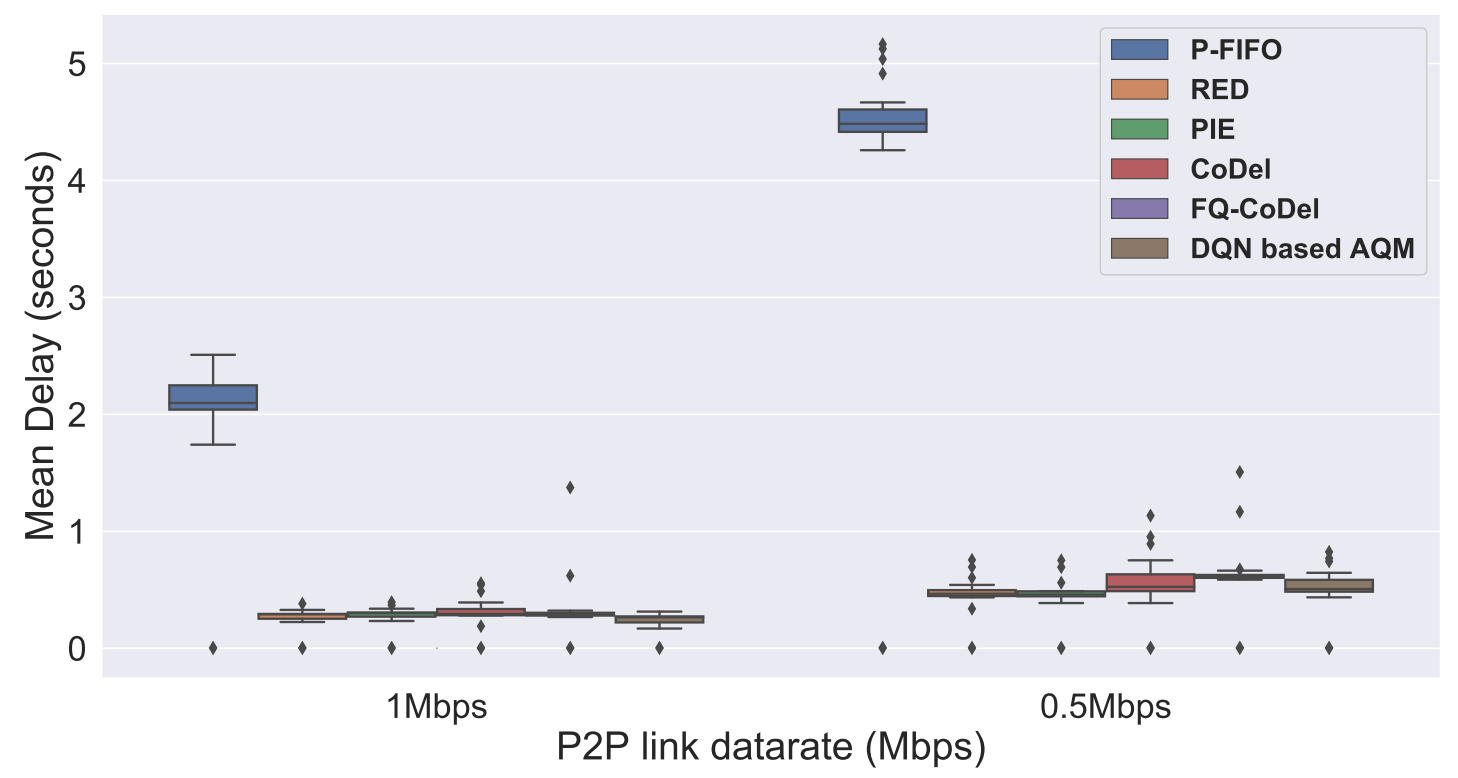

Figure 5.4: Mean delay per flows of 21 IoT devices; Baseline RTT $=0 \mathrm{~ms}$; Reward scaling factor $\delta=0.5$ 
and outperforms other AQM schemes in terms of queuing delay on all given cases except for $50 \mathrm{~ms}$ of RTT in that our approach is delay-aware learning algorithm. And generally, queuing delay of AQM schemes are not affected by different RTTs a lot on each delay-related figures.

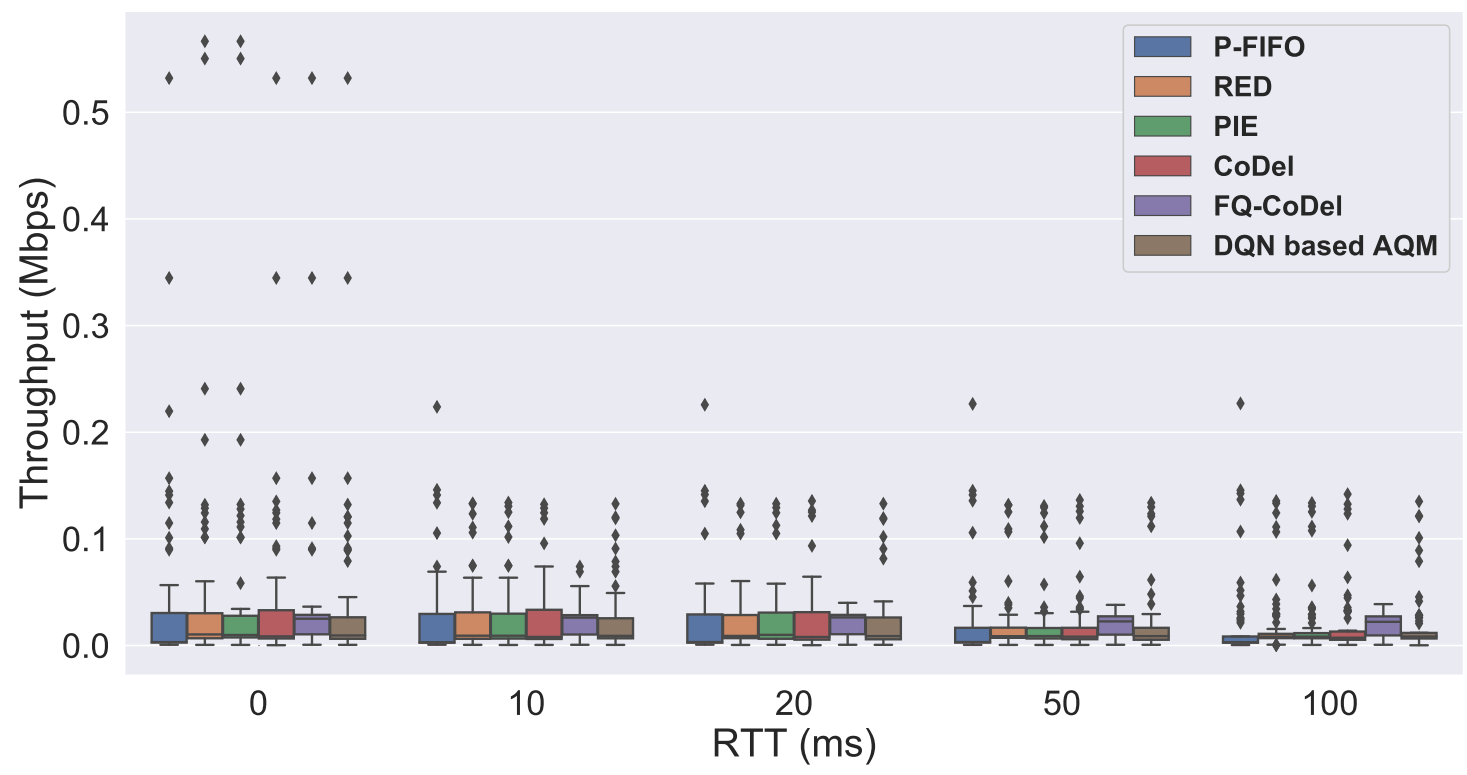

Figure 5.5: Throughput per flows of 63 IoT devices; P2P link data rate $=1 \mathrm{Mbps}$; Reward scaling factor $\delta=0.5$

\subsection{Analysis on Different IoT Categories}

Depending on the IoT categories, we set different transport layer protocols. For smart cameras, we set UDP protocol assuming the real-time monitoring and surveillance and store streaming data into cloud servers. For other categories consisting of Hubs, Switches \& Triggers, Air quality sensors, Healthcare devices, Light bulbs, and Electronics, we set TCP protocol assuming HTTPS protocol which is mostly used for IoT devices in [33] and the reliable communication. 


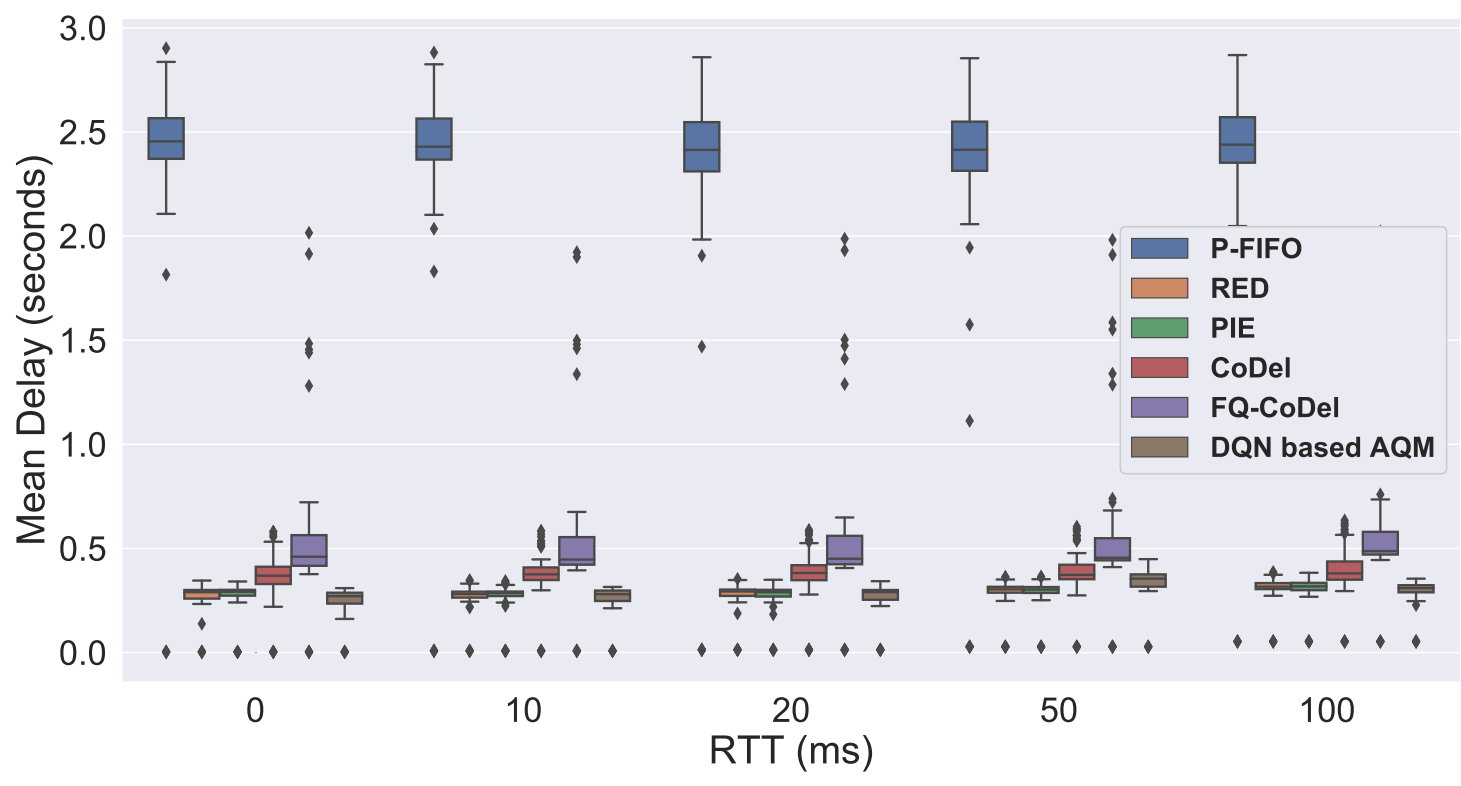

Figure 5.6: Mean delay per flows of 63 IoT devices; P2P link data rate $=1 \mathrm{Mbps}$; Reward scaling factor $\delta=0.5$

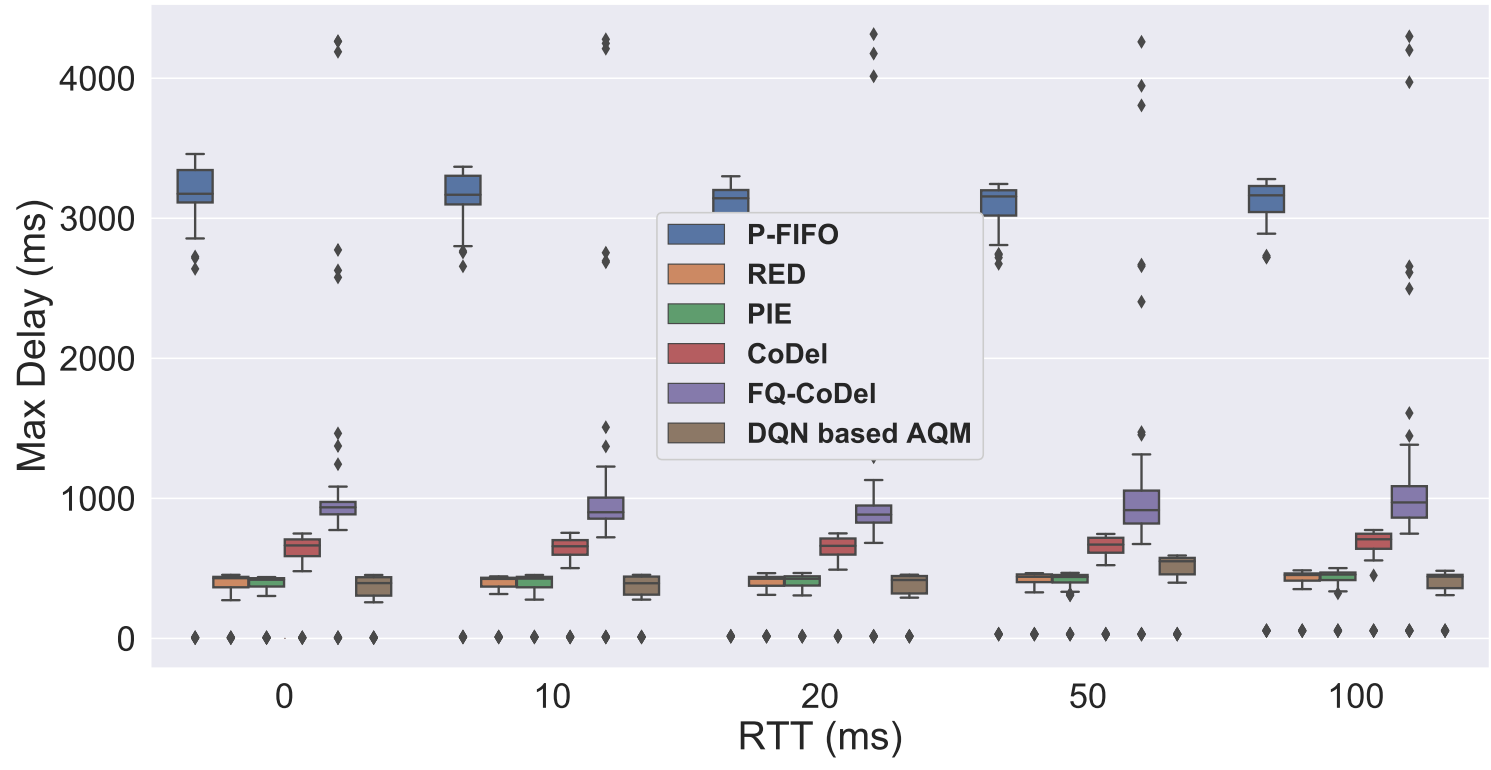

Figure 5.7: Maximum delay per flows of 63 IoT devices; P2P link data rate $=1 \mathrm{Mbps}$; Reward scaling factor $\delta=0.5$ 


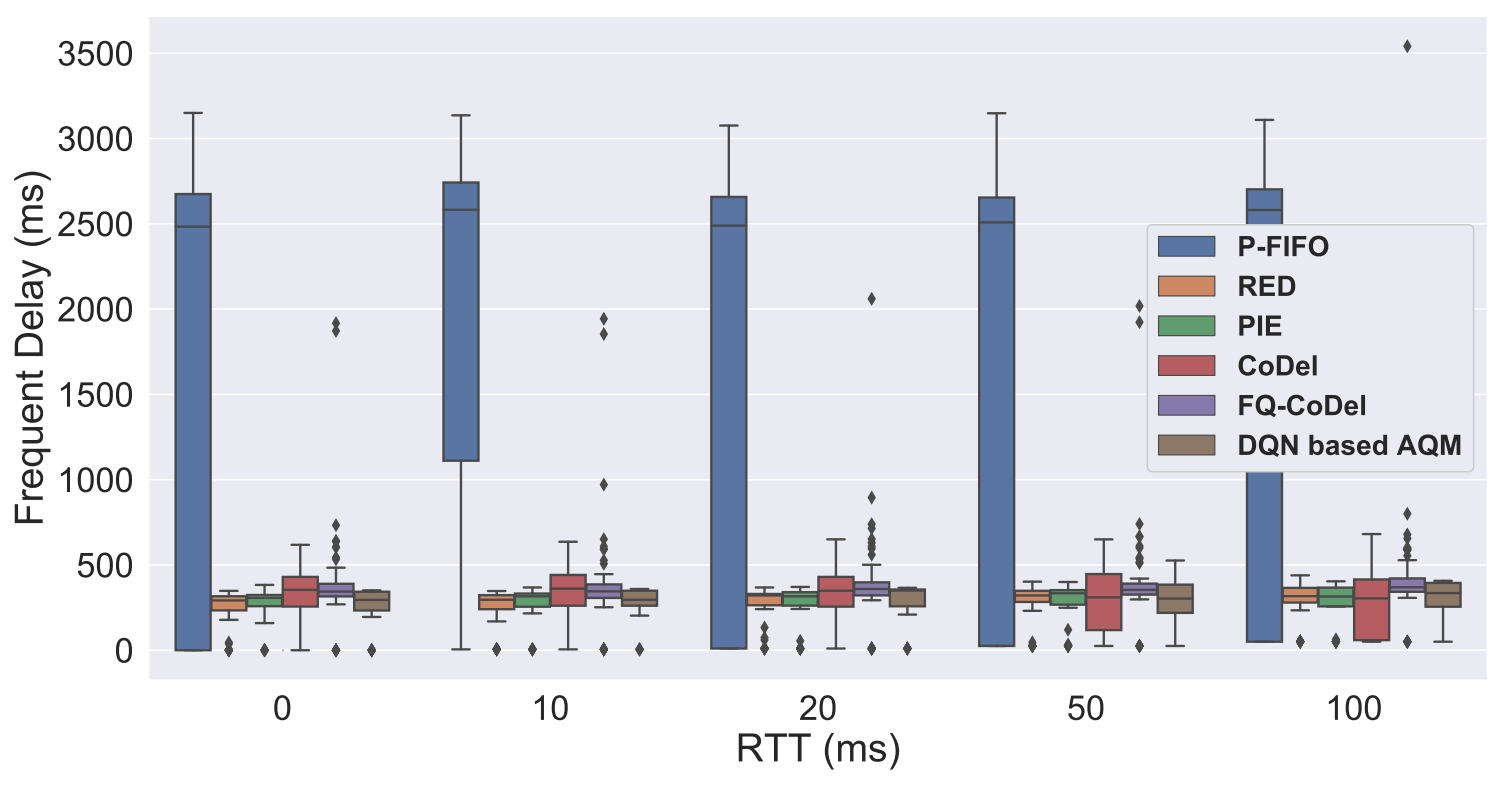

Figure 5.8: Most frequent delay per flows of 63 IoT devices; P2P link data rate $=1 \mathrm{Mbps}$; Reward scaling factor $\delta=0.5$

\subsubsection{UDP based IoT flows}

We classify UDP based IoT flows generated by smart cameras from the experiment of section 5.3, and Figures 5.9, 5.10, and 5.11 are boxplots of throughput, mean delay, and mean jitter per UDP flows respectively. In previous experimental results, FQ-CoDel has shown fine performance in terms of throughput in boxplots, however considering only UDP flows, FQ-CoDel shows deficient performances on all testbeds and metrics. In order to measure QoS to end users in networking, jitter is also an useful metrics for UDP based real-time communication such as video streaming and Voice over IP (VoIP). In Figure 5.11, we can observe that DQN based AQM sustains the low jitter regardless of RTTs.

\subsubsection{TCP based IoT flows}

TCP based packet transmission is one of the main reasons that causes traffic congestion due to the three-way handshake referred to "SYN-SYN-ACK". On this testbed, Figures 


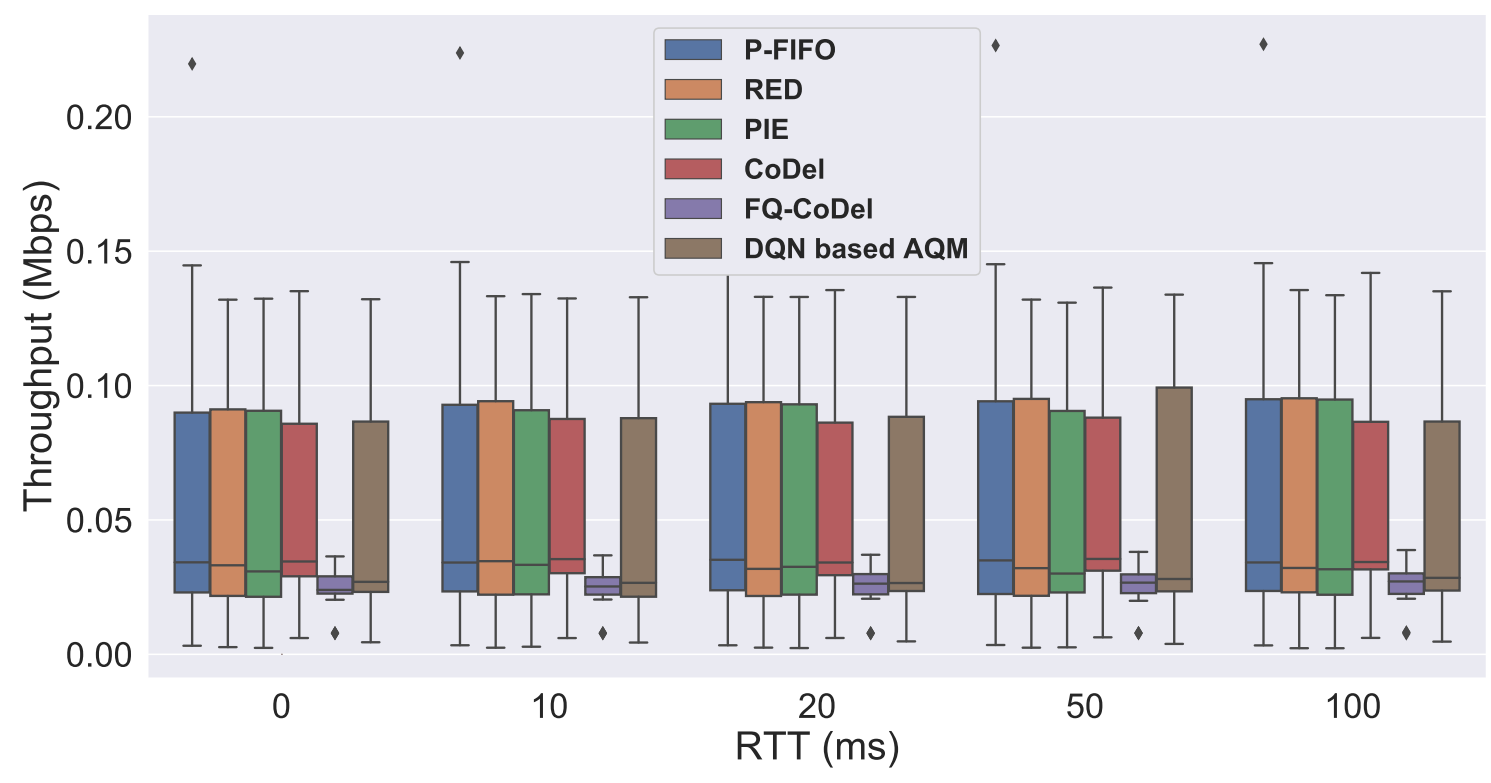

Figure 5.9: Throughput per UDP flows of 18 IoT devices; P2P link data rate = 1Mbps; Reward scaling factor $\delta=0.5$

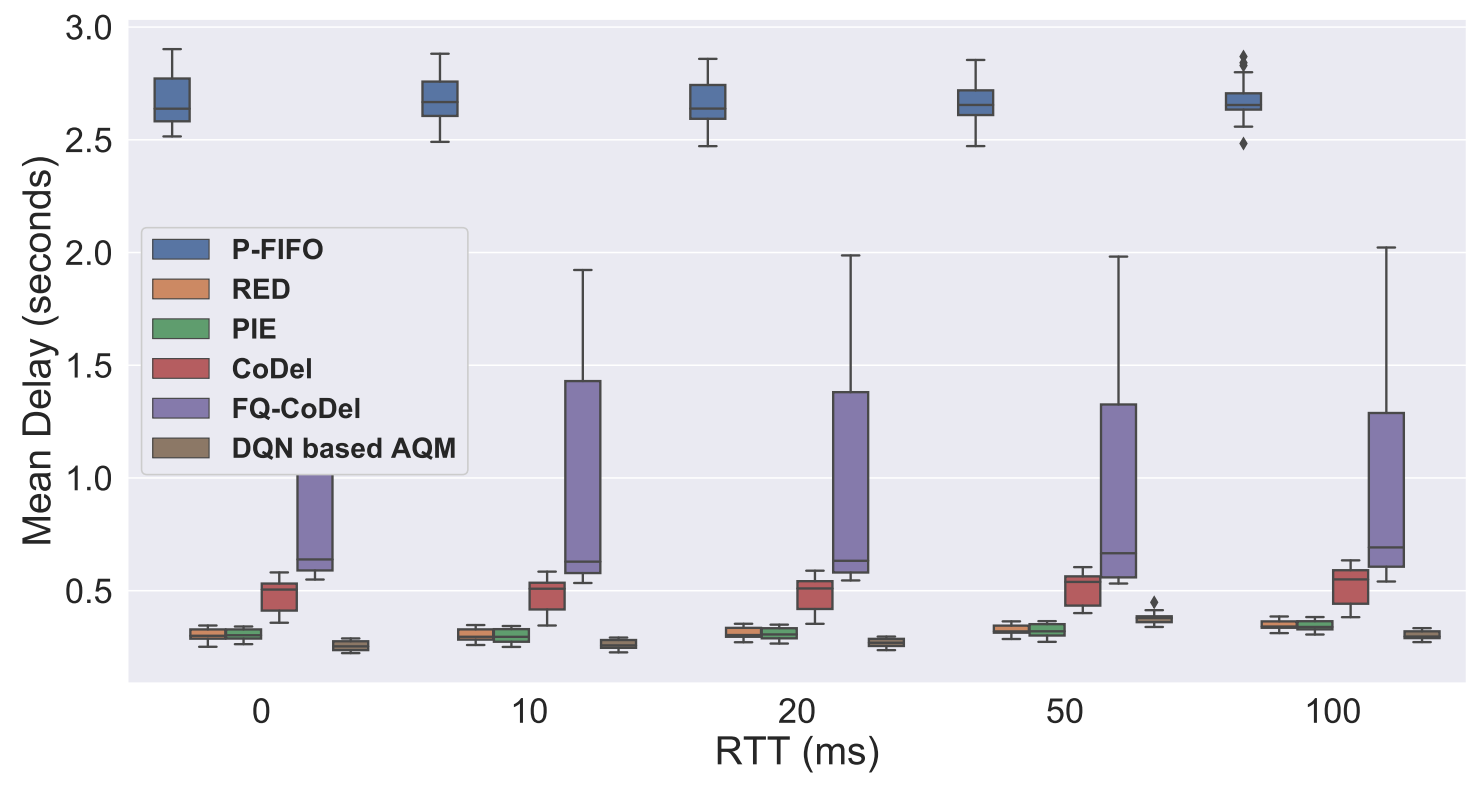

Figure 5.10: Mean Delay per UDP flows of 18 IoT devices; P2P link data rate = 1Mbps; Reward scaling factor $\delta=0.5$ 


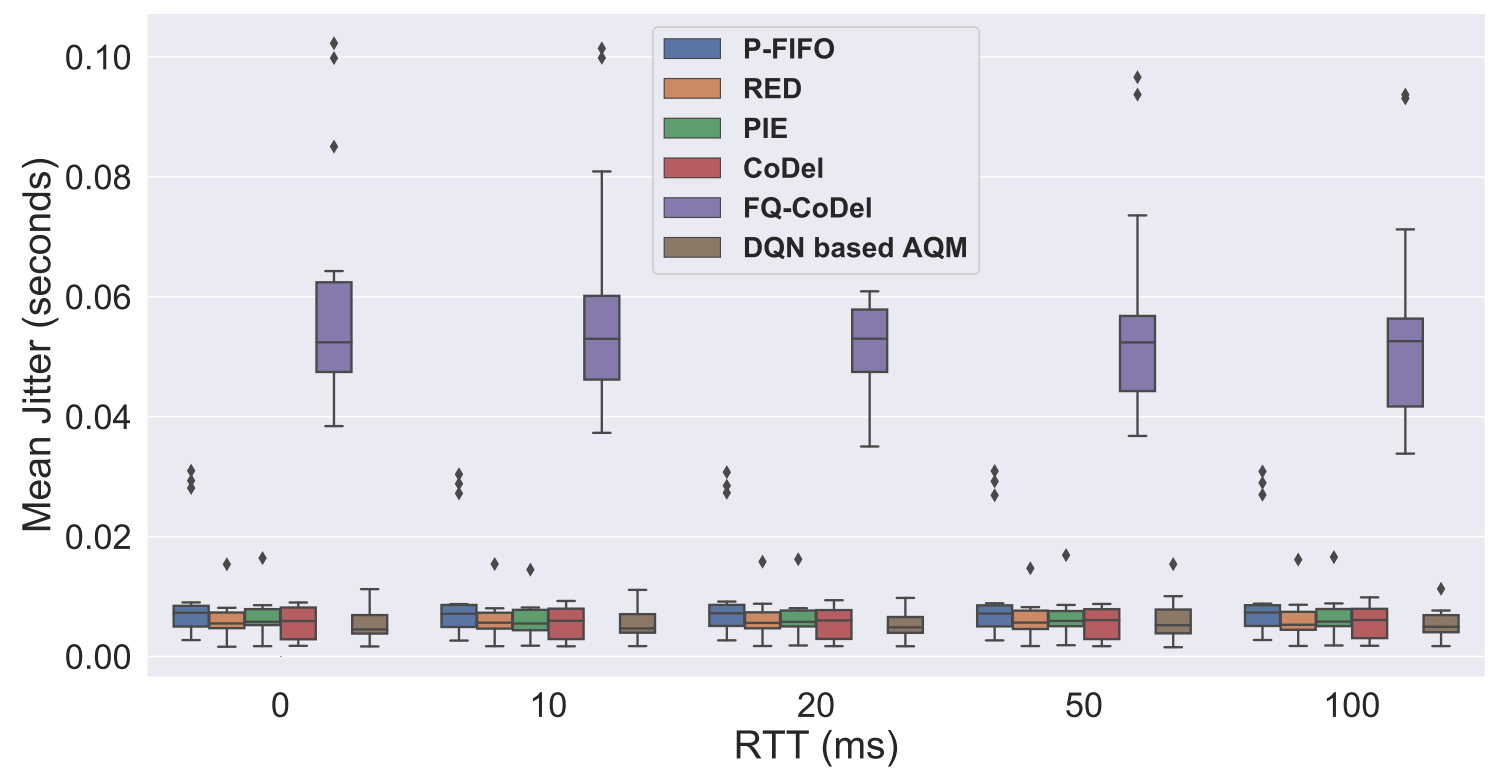

Figure 5.11: Mean Jitter per UDP flows of 18 IoT devices; P2P link data rate $=1 \mathrm{Mbps}$; Reward scaling factor $\delta=0.5$

5.12 and 5.13 show the throughput and delay on 48 TCP flows of IoT devices. Unlike UDP flows, FQ-CoDel shows better throughput and DQN based AQM's throughput is analogous to other AQM schemes. But with regard to the delay, DQN based AQM shows notable performance as compared with targeted AQM schemes.

\subsection{Analysis on Reward Trajectory for Model Opti- mization}

In this section, we focus on our reward function and how it effects to the results. Figure 5.14 shows the comparison of rewards on non-trained/trained network model, and we can see that cumulative reward of the trained model is much higher than that of the non-trained model. However, the non-trained model's cumulative reward increases linearly as well, since it is being trained in parallel through the simulation time step. In Figure 5.14(b), non- 


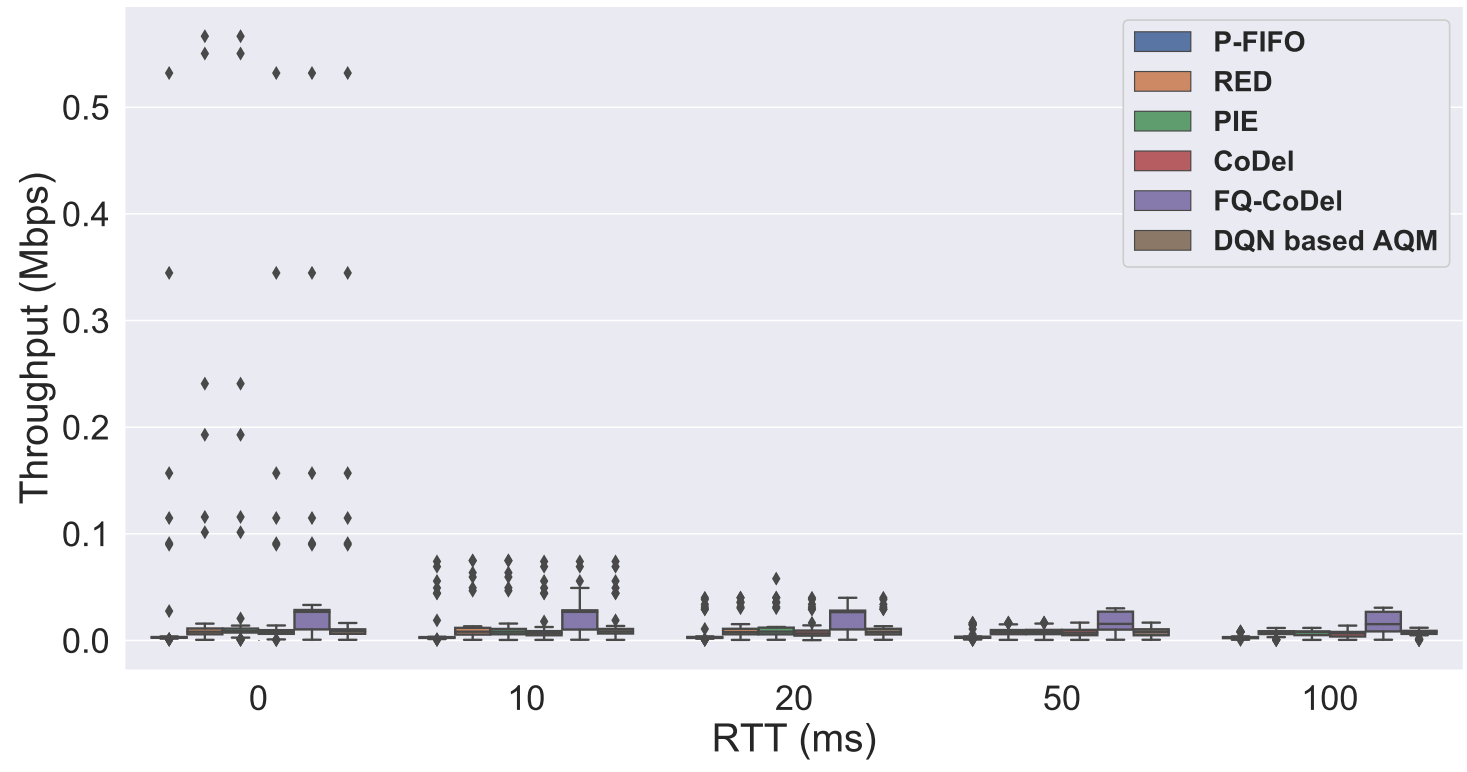

Figure 5.12: Throughput per TCP flows of 45 IoT devices; P2P link data rate = 1Mbps; Reward scaling factor $\delta=0.5$

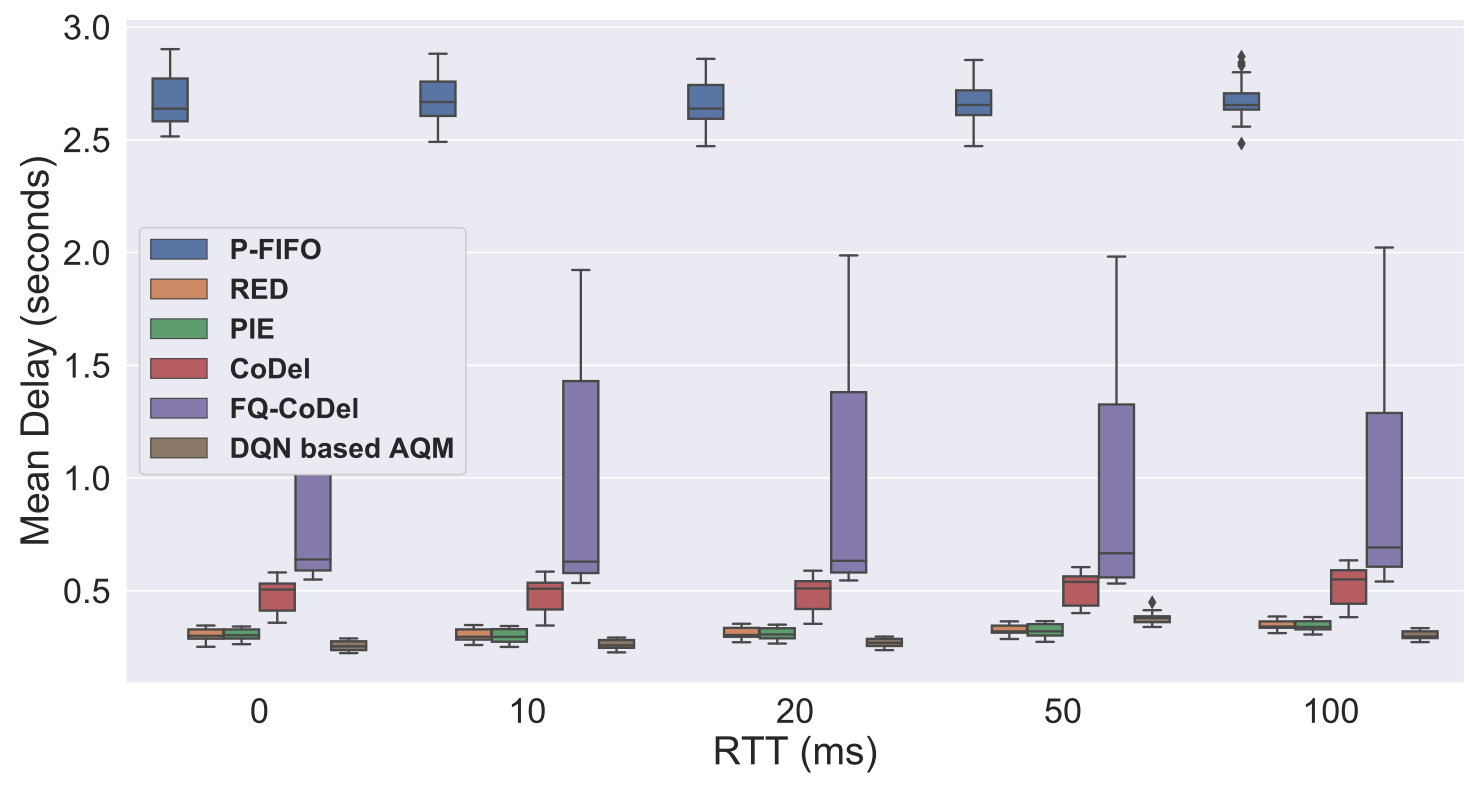

Figure 5.13: Mean Delay per TCP flows of 45 IoT devices; P2P link data rate = 1Mbps; Reward scaling factor $\delta=0.5$ 


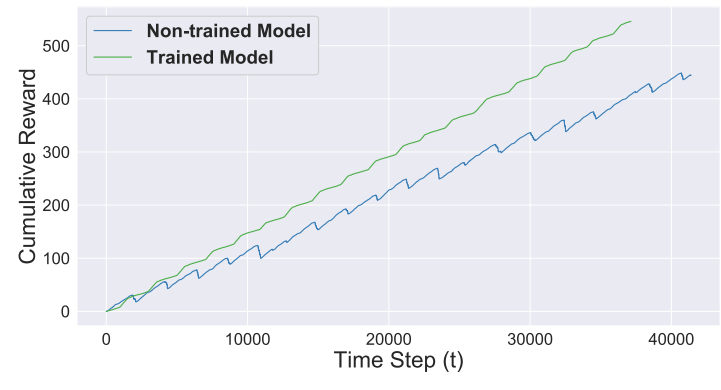

(a) Cumulative reward

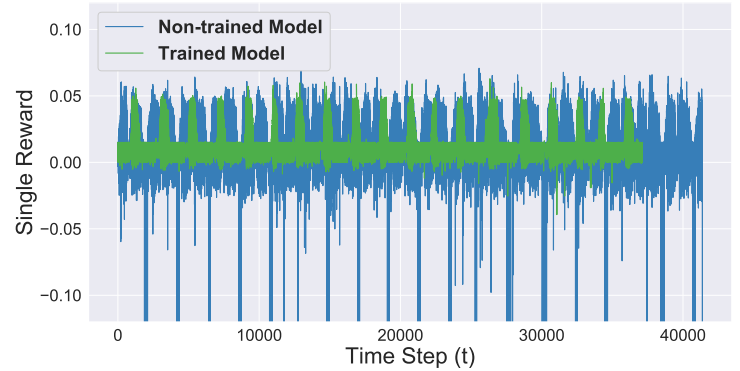

(b) Single reward

Figure 5.14: Comparison of reward on non-trained/trained model; P2P link data rate $=$ 1Mbps; Baseline RTT $=0 \mathrm{~ms}$; Number of IoT devices $=63$; Reward scaling factor $\delta=0.5$

trained model shows the unstable behaviour because its action has randomness. Comparing with the non-trained model, trained model performs $22.362 \mathrm{~ms}$ and $0.57841 \mathrm{~ms}$ shorter in delay and jitter respectively. Table 5.1 tabulates the sensitivity depending on the reward scaling factor $\delta$. Higher scaling factor stands for that the reward is more weighted towards delay_reward than enqueue_reward by the agent. Since we use drop-tail function when the queue is filled with packets, packets are dropped by either exceeded queue size or packetdropping action, and that is why the packet drop-rate is not in inversely proportion of the mean delay. However, we can also observe that depending on the higher scaling factor, mean delay is decreased.

Table 5.1: Reward scaling factor sensitivity; P2P link data rate $=1 \mathrm{Mbps}$; Baseline $\mathrm{RTT}=$ 0ms; Number of IoT devices $=63$

\begin{tabular}{|c|c|c|c|c|}
\hline Scaling factor & Throughput & Packet drop-rate & Mean delay & Mean jitter \\
\hline 0.4 & $0.954097 \mathrm{Mbps}$ & $24.23 \%$ & $0.267 \mathrm{~s}$ & $0.008 \mathrm{~s}$ \\
\hline 0.5 & $0.953510 \mathrm{Mbps}$ & $24.17 \%$ & $0.197 \mathrm{~s}$ & $0.007 \mathrm{~s}$ \\
\hline 0.6 & $0.954050 \mathrm{Mbps}$ & $23.1 \%$ & $0.195 \mathrm{~s}$ & $0.0068 \mathrm{~s}$ \\
\hline
\end{tabular}




\subsection{Summary}

In this chapter, we analyzed the experimental results on different testbeds. In general, PFIFO performed inferior since the P-FIFO enabled queue works passively, and FQ-CoDel

showed fine throughput performance, but suffers from the trade-off with high delay. The proposed DQN based AQM scheme verified low queuing delay on most cases preserving above the average throughput. In summary, Appendix B tabulates average throughput, packet drop-rate, delay, and jitter for each AQM schemes in various experimental environments, and DQN based AQM performs competitively trading off between mean delay and throughput, as well as low jitter. In the next chapter, we conclude this thesis by providing overall summary with future work to enhance efficient network management in IoT based networks. 


\section{Chapter 6}

\section{Conclusion}

\subsection{Conclusion and Summary}

In this thesis, we proposed the design of Deep Reinforcement Learning based Active Queue Management. As a baseline model of the design, we selected Deep Q-Network since the state transition in networking is discrete and it is able to be expressed as a finite Markov Decision Process which is the fundamental principle of reinforcement learning. From the state setup to the reward function design, we implemented them on $n s$-3 network simulator by applying deep learning framework tiny-dnn. Along with fog/edge computing architecture, we also constructed the IoT testbed by reflecting real IoT devices' characteristics including periodic operating cycle, actual transmission rate, packet size, and peak rate in wireless communication environment. We deployed the AQM scheme at the interface of the fog/edge device connected to the cloud gateway, and our proposed scheme achieved substantial performances such as low queuing delay and jitter on the stochastic IoT environment simultaneously maintaining good throughput comparing with widely used AQM schemes. 


\subsection{Future Work}

\subsubsection{Energy Efficient AQM Design}

Although the fog/edge device has sufficient computation capability, energy efficient design plays a significant role for ideal future Internet infrastructure due to the fact that the number of devices of tier-1 is increasing exponentially in our life. In terms of energy efficient AQM, we expect that it can be achieved by increasing the interval of packet-drop probability calculation and optimizing memory usage. For DL based AQM, the light neural network model such as Binarized Neural Network (BNN) [39] can be used for the energy efficient AQM design. And applying optimal model and tuning hyper-parameters are always huge challenges for deep learning applied work. Deep learning field is growing extremely fast, and performance of the algorithms are being improved day by day. Optimal model can be achieved using model compression techniques such as weight pruning. Hyper-parameter tuning of selected model is also capable of improving the performance as well as selecting a good model, and even it has been extended as a new field which is Automatic Machine Learning (AutoML).

\subsubsection{Construction of IoT-realistic Simulation Environment}

In our experiment, we used IEEE $802.11 \mathrm{n} 5 \mathrm{GHz}$ as a Wi-Fi standard, and TCP and UDP protocols for transmission. If we set lightweight and resource limited protocol such as Constrained Application Protocol (CoAP) and message queue telemetry transport (MQTT) on the 6LoWPAN which is designed for low power and narrow bandwidth for IoT specified by IEEE 802.15.4, we would conduct more ideal experiments and get more accurate results [3]. 


\section{Appendix A}

\section{Dequeue Rate Calculation in PIE}

1. Upon packet departure, decide to be in a measurement cycle if :

$$
\text { qlen >dq_threshold; }
$$

2. If the above is true, update the departure count in bytes $d q_{-}$count :

$$
d q_{-} \text {count }=d q_{\_} \text {count }+d q \_p k t s i z e ;
$$

3. Update dequeue rate once $d q_{-}$count $>d q_{-}$threshold with averaging parameter $\epsilon$, set to 0.5 by default, and reset counters :

$$
\begin{gathered}
d q \_i n t=\text { time }_{\text {now }}-\text { time }_{\text {start }} ; \\
d q \_r a t e=\frac{d q \_c o u n t}{d q \_i n t} ; \\
\text { avg_drate }=(1-\epsilon) * \text { avg_drate }+\epsilon * d q \_r a t e \\
t_{\text {ime }} \text { start }=t i m e_{\text {now }} . \\
d d_{-} \text {count }=0 ;
\end{gathered}
$$




\section{Appendix B}

\section{AQM Performance Comparison in Various Environments}

Table B.1: Performance comparison for all flows of 21 IoT devices; P2P link data rate $=$ $1 \mathrm{Mbps}$; Baseline RTT $=0 \mathrm{~ms}$; Reward scaling factor $\delta=0.6$.

\begin{tabular}{|c|c|c|c|c|}
\hline AQM scheme & Total throughput & Packet drop-rate & Mean delay & Mean jitter \\
\hline P-FIFO & $0.9537 \mathrm{Mbps}$ & $5.36 \%$ & $1.736 \mathrm{~s}$ & $0.0192 \mathrm{~s}$ \\
\hline RED & $0.9512 \mathrm{Mbps}$ & $23 \%$ & $0.222 \mathrm{~s}$ & $0.0073 \mathrm{~s}$ \\
\hline PIE & $0.9513 \mathrm{Mbps}$ & $23.79 \%$ & $0.23 \mathrm{~s}$ & $0.0072 \mathrm{~s}$ \\
\hline CoDel & $0.9539 \mathrm{Mbps}$ & $18.81 \%$ & $0.284 \mathrm{~s}$ & $0.0099 \mathrm{~s}$ \\
\hline FQ-CoDel & $0.9538 \mathrm{Mbps}$ & $18.72 \%$ & $0.379 \mathrm{~s}$ & $0.0151 \mathrm{~s}$ \\
\hline DQN based AQM & $\mathbf{0 . 9 5 4 1 M b p s}$ & $\mathbf{2 3 . 1 \%}$ & $\mathbf{0 . 1 9 5 s}$ & $\mathbf{0 . 0 0 6 8 s}$ \\
\hline
\end{tabular}


Table B.2: Performance comparison for all flows of 21 IoT devices; P2P link data rate $=$ $0.5 \mathrm{Mbps}$; Baseline RTT $=0 \mathrm{~ms}$; Reward scaling factor $\delta=0.5$.

\begin{tabular}{|c|c|c|c|c|}
\hline AQM scheme & Total throughput & Packet drop-rate & Mean delay & Mean jitter \\
\hline P-FIFO & $0.4766 \mathrm{Mbps}$ & $17.24 \%$ & $4.69 \mathrm{~s}$ & $0.0329 \mathrm{~s}$ \\
\hline RED & $0.4755 \mathrm{Mbps}$ & $43.3 \%$ & $0.5326 \mathrm{~s}$ & $0.0174 \mathrm{~s}$ \\
\hline PIE & $0.4755 \mathrm{Mbps}$ & $43.1 \%$ & $0.5231 \mathrm{~s}$ & $0.0179 \mathrm{~s}$ \\
\hline CoDel & $0.4767 \mathrm{Mbps}$ & $36.52 \%$ & $0.7242 \mathrm{~s}$ & $0.0228 \mathrm{~s}$ \\
\hline FQ-CoDel & $0.4769 \mathrm{Mbps}$ & $44.55 \%$ & $0.7997 \mathrm{~s}$ & $0.032 \mathrm{~s}$ \\
\hline DQN based AQM & $\mathbf{0 . 4 7 6 7 M b p s}$ & $\mathbf{3 9 . 9 4 \%}$ & $\mathbf{0 . 5 9 1 9 s}$ & $\mathbf{0 . 0 1 8 5}$ \\
\hline
\end{tabular}

Table B.3: Performance comparison for all flows of 63 IoT devices; P2P link data rate $=$ $1 \mathrm{Mbps}$; Baseline RTT $=0 \mathrm{~ms}$; Reward scaling factor $\delta=0.5$.

\begin{tabular}{|c|c|c|c|c|}
\hline AQM scheme & Total throughput & Packet drop-rate & Mean delay & Mean jitter \\
\hline P-FIFO & $0.9535 \mathrm{Mbps}$ & $46.97 \%$ & $2.1559 \mathrm{~s}$ & $0.0245 \mathrm{~s}$ \\
\hline RED & $0.9524 \mathrm{Mbps}$ & $53.72 \%$ & $0.2944 \mathrm{~s}$ & $0.0092 \mathrm{~s}$ \\
\hline PIE & $0.9524 \mathrm{Mbps}$ & $54.24 \%$ & $0.3044 \mathrm{~s}$ & $0.095 \mathrm{~s}$ \\
\hline CoDel & $0.9535 \mathrm{Mbps}$ & $50.5 \%$ & $0.3712 \mathrm{~s}$ & $0.0103 \mathrm{~s}$ \\
\hline FQ-CoDel & $0.9543 \mathrm{Mbps}$ & $63 \%$ & $0.5015 \mathrm{~s}$ & $0.0707 \mathrm{~s}$ \\
\hline DQN based AQM & $\mathbf{0 . 9 5 3 8 M b p s}$ & $\mathbf{5 4 . 0 4 \%}$ & $\mathbf{0 . 2 7 0 9 s}$ & $\mathbf{0 . 0 0 7 6 s}$ \\
\hline
\end{tabular}

Table B.4: Performance comparison for all flows of 63 IoT devices; P2P link data rate = $1 \mathrm{Mbps}$; Baseline RTT $=10 \mathrm{~ms}$; Reward scaling factor $\delta=0.5$.

\begin{tabular}{|c|c|c|c|c|}
\hline AQM scheme & Total throughput & Packet drop-rate & Mean delay & Mean jitter \\
\hline P-FIFO & $0.9532 \mathrm{Mbps}$ & $46.5 \%$ & $2.616 \mathrm{~s}$ & $0.0238 \mathrm{~s}$ \\
\hline RED & $0.9522 \mathrm{Mbps}$ & $53.35 \%$ & $0.3065 \mathrm{~s}$ & $0.0093 \mathrm{~s}$ \\
\hline PIE & $0.9522 \mathrm{Mbps}$ & $53.55 \%$ & $0.3067 \mathrm{~s}$ & $0.0093 \mathrm{~s}$ \\
\hline CoDel & $0.9532 \mathrm{Mbps}$ & $50.41 \%$ & $0.4754 \mathrm{~s}$ & $0.0104 \mathrm{~s}$ \\
\hline FQ-CoDel & $0.954 \mathrm{Mbps}$ & $63.03 \%$ & $0.7458 \mathrm{~s}$ & $0.071 \mathrm{~s}$ \\
\hline DQN based AQM & $\mathbf{0 . 9 5 3 4 M b p s}$ & $\mathbf{5 3 . 9 9 \%}$ & $\mathbf{0 . 2 7 7 3 s}$ & $\mathbf{0 . 0 0 7 6 s}$ \\
\hline
\end{tabular}


Table B.5: Performance comparison for all flows of 63 IoT devices; P2P link data rate $=$ 1Mbps; Baseline RTT $=20 \mathrm{~ms}$; Reward scaling factor $\delta=0.5$.

\begin{tabular}{|c|c|c|c|c|}
\hline AQM scheme & Total throughput & Packet drop-rate & Mean delay & Mean jitter \\
\hline P-FIFO & $0.9527 \mathrm{Mbps}$ & $46.04 \%$ & $2.601 \mathrm{~s}$ & $0.0238 \mathrm{~s}$ \\
\hline RED & $0.9521 \mathrm{Mbps}$ & $53.54 \%$ & $0.3138 \mathrm{~s}$ & $0.0094 \mathrm{~s}$ \\
\hline PIE & $0.9521 \mathrm{Mbps}$ & $53.6 \%$ & $0.3142 \mathrm{~s}$ & $0.0094 \mathrm{~s}$ \\
\hline CoDel & $0.9519 \mathrm{Mbps}$ & $50.22 \%$ & $0.4784 \mathrm{~s}$ & $0.0102 \mathrm{~s}$ \\
\hline FQ-CoDel & $0.9535 \mathrm{Mbps}$ & $62.74 \%$ & $0.7493 \mathrm{~s}$ & $0.07 \mathrm{~s}$ \\
\hline DQN based AQM & $\mathbf{0 . 9 5 3 M b p s}$ & $\mathbf{5 4 . 1 4 \%}$ & $\mathbf{0 . 2 8 3 1 s}$ & $\mathbf{0 . 0 0 7 8 s}$ \\
\hline
\end{tabular}

Table B.6: Performance comparison for all flows of 63 IoT devices; P2P link data rate = $1 \mathrm{Mbps}$; Baseline RTT $=50 \mathrm{~ms}$; Reward scaling factor $\delta=0.5$.

\begin{tabular}{|c|c|c|c|c|}
\hline AQM scheme & Total throughput & Packet drop-rate & Mean delay & Mean jitter \\
\hline P-FIFO & $0.9522 \mathrm{Mbps}$ & $45.86 \%$ & $2.604 \mathrm{~s}$ & $0.0236 \mathrm{~s}$ \\
\hline RED & $0.9503 \mathrm{Mbps}$ & $53.41 \%$ & $0.3279 \mathrm{~s}$ & $0.0093 \mathrm{~s}$ \\
\hline PIE & $0.9516 \mathrm{Mbps}$ & $53.87 \%$ & $0.3286 \mathrm{~s}$ & $0.01 \mathrm{~s}$ \\
\hline CoDel & $0.9513 \mathrm{Mbps}$ & $49.89 \%$ & $0.4908 \mathrm{~s}$ & $0.0104 \mathrm{~s}$ \\
\hline FQ-CoDel & $0.9531 \mathrm{Mbps}$ & $62.6 \%$ & $0.7702 \mathrm{~s}$ & $0.0702 \mathrm{~s}$ \\
\hline DQN based AQM & $\mathbf{0 . 9 5 1 8 M b p s}$ & $\mathbf{5 3 . 1 \%}$ & $\mathbf{0 . 3 7 8 1} \mathbf{s}$ & $\mathbf{0 . 0 1 0 2 s}$ \\
\hline
\end{tabular}

Table B.7: Performance comparison for all flows of 63 IoT devices; P2P link data rate $=$ 1Mbps; Baseline RTT $=100 \mathrm{~ms}$; Reward scaling factor $\delta=0.5$.

\begin{tabular}{|c|c|c|c|c|}
\hline AQM scheme & Total throughput & Packet drop-rate & Mean delay & Mean jitter \\
\hline P-FIFO & $0.9518 \mathrm{Mbps}$ & $45.67 \%$ & $2.621 \mathrm{~s}$ & $0.0234 \mathrm{~s}$ \\
\hline RED & $0.9492 \mathrm{Mbps}$ & $53.11 \%$ & $0.3487 \mathrm{~s}$ & $0.0098 \mathrm{~s}$ \\
\hline PIE & $0.9495 \mathrm{Mbps}$ & $53.49 \%$ & $0.3473 \mathrm{~s}$ & $0.0101 \mathrm{~s}$ \\
\hline CoDel & $0.9455 \mathrm{Mbps}$ & $49.48 \%$ & $0.509 \mathrm{~s}$ & $0.0107 \mathrm{~s}$ \\
\hline FQ-CoDel & $0.9526 \mathrm{Mbps}$ & $62.38 \%$ & $0.7957 \mathrm{~s}$ & $0.0714 \mathrm{~s}$ \\
\hline DQN based AQM & $\mathbf{0 . 9 5 1 6 M b p s}$ & $\mathbf{5 4 . 0 6 \%}$ & $\mathbf{0 . 3 1 5 5 s}$ & $\mathbf{0 . 0 0 8 6 s}$ \\
\hline
\end{tabular}




\section{Bibliography}

[1] IDC, "Worldwide Internet of Things Forecast Update, 20172021," in document US43304017, IDC, February 2018. [Online]. Available: https://www.idc.com/getdoc.jsp?containerId=US43304017.

[2] Gartner, "Gartner says a typical family home could contain more than 500 smart devices by 2022," September 2014. [Online]. Available: https://www.gartner.com/newsroom/id/2839717.

[3] J. Lin, W. Yu, N. Zhang, X. Yang, H. Zhang, and W. Zhao, "A Survey on Internet of Things: Architecture, Enabling Technologies, Security and Privacy, and Applications," IEEE Internet of Things Journal, vol. 4, pp. 1125-1142, March 2017. [Online]. Available: https://doi.org/10.1109/JIOT.2017.2683200.

[4] M. Mukherjee, L. Shu, and D. Wang, "Survey of Fog Computing: Fundamental, Network Applications, and Research Challenges," IEEE Communications Surveys Tutorials, vol. 20, pp. 1826-1857, March 2018. [Online]. Available: https://doi.org/10.1109/COMST.2018.2814571.

[5] N. Kuhn, P. Natarajan, N. Khademi, and D. Ros, "Characterization Guidelines for Active Queue Management (AQM)," in Internet Engineering Task Force (IETF), RFC 7928, July 2016. [Online]. Available: https://tools.ietf.org/html/rfc7928. 
[6] F. Baker and G. Fairhurst, "IETF Recommendations Regarding Active Queue Management," in Internet Engineering Task Force (IETF), RFC 7567, July 2015. [Online]. Available: https://tools.ietf.org/html/rfc7567.

[7] M. Mohammadi, A. Al-Fuqaha, S. Sorour, and M. Guizani, "Deep Learning for IoT Big Data and Streaming Analytics: A Survey," IEEE Communications Surveys Tutorials, June 2018. [Online early access]. Available: https://doi.org/10.1109/COMST.2018.2844341.

[8] R. S. Sutton and A. G. Barto, Reinforcement Learning: An Introduction. MIT Press, second ed., 2018.

[9] N. C. Luong, D. T. Hoang, S. Gong, D. Niyato, P. Wang, Y.-C. Liang, and D. I. Kim, "Applications of Deep Reinforcement Learning in Communications and Networking: A Survey," in arXiv preprint arXiv:1810.07862, October 2018. [Online]. Available: https://arxiv.org/abs/1810.07862.

[10] S. Floyd and V. Jacobson, "Random Early Detection Gateways for Congestion Avoidance," IEEE/ACM Transactions on Networking, vol. 1, pp. 397-413, August 1993. [Online]. Available: https://doi.org/10.1109/90.251892.

[11] K. Nichols and V. Jacobson, "Controlling Queue Delay," Communications of the ACM, vol. 55, pp. 42-50, July 2012. [Online]. Available: https://doi.org/10.1145/2209249.2209264.

[12] R. Pan, P. Natarajan, C. Piglione, M. S. Prabhu, V. Subramanian, F. Baker, and B. VerSteeg, "PIE: A lightweight control scheme to address the bufferbloat problem," in 2013 IEEE 14th International Conference on High Performance Switching and Routing (HPSR), July 2013. [Online]. Available: https://doi.org/10.1109/HPSR.2013.6602305. 
[13] A. Sungur, "Tcp - random early detection (red) mechanism for congestion control," Master's thesis, Rochester Institute of Technology, 2015. [Online]. Available: https://scholarworks.rit.edu/theses/8904.

[14] T. Hoeiland-Joergensen, P. McKenney, D. Taht, J. Gettys, and E. Dumazet, "The Flow Queue CoDel Packet Scheduler and Active Queue Management Algorithm," in Internet Engineering Task Force (IETF), RFC 8290, January 2018. [Online]. Available: https://tools.ietf.org/html/rfc8290.

[15] N. Bouacida and B. Shihada, "Practical and Dynamic Buffer Sizing using LearnQueue," IEEE Transactions on Mobile Computing, September 2018. [Online early access]. Available: https://doi.org/10.1109/TMC.2018.2868670.

[16] S. K. Bisoy, P. K. Pandey, and B. Pati, "Design of an active queue management technique based on neural networks for congestion control," in 2017 IEEE International Conference on Advanced Networks and Telecommunications Systems (ANTS), December 2017. [Online]. Available: https://doi.org/10.1109/ANTS.2017.8384104.

[17] N. Vucevic, J. Perez-Romero, O. Sallent, and R. Agusti, "Reinforcement Learning for Active Queue Management in Mobile All-IP Networks," in 2007 IEEE 18th International Symposium on Personal, Indoor and Mobile Radio Communications, September 2007. [Online]. Available: https://doi.org/10.1109/PIMRC.2007.4394713.

[18] X. Chen, H. Zhang, C. Wu, S. Mao, Y. Ji, and M. Bennis, "Optimized Computation Offloading Performance in Virtual Edge Computing Systems via Deep Reinforcement Learning," IEEE Internet of Things Journal, October 2018. [Online early access]. Available: https://doi.org/10.1109/JIOT.2018.2876279.

[19] S. Chinchali, P. Hu, T. Chu, M. Sharma, M. Bansal, R. Misra, M. Pavone, and S. Katti, "Cellular Network Traffic Scheduling With Deep Reinforcement Learning," in Thirty- 
Second AAAI Conference on Artificial Intelligence, February 2018. [Online]. Available: https://www.aaai.org/ocs/index.php/AAAI/AAAI18/paper/view/16638.

[20] Z. Xu, J. Tang, J. Meng, W. Zhang, Y. Wang, C. H. Liu, and D. Yang, "Experiencedriven Networking: A Deep Reinforcement Learning based Approach," in IEEE INFOCOM 2018 - IEEE Conference on Computer Communications, April 2018. [Online]. Available: https://doi.org/10.1109/INFOCOM.2018.8485853.

[21] J. Kua, S. H. Nguyen, G. Armitage, and P. Branch, "Using Active Queue Management to Assist IoT Application Flows in Home Broadband Networks," IEEE Internet of Things Journal, vol. 4, pp. 1399-1407, July 2017. [Online]. Available: https://doi.org/10.1109/JIOT.2017.2722683.

[22] Y. Zhang, B. Feng, W. Quan, G. Li, H. Zhou, and H. Zhang, "Theoretical Analysis on Edge Computation Offloading Policies for IoT Devices," IEEE Internet of Things Journal, October 2018. [Online early access]. Available: https://doi.org/10.1109/JIOT.2018.2875599.

[23] A. Yousefpour, G. Ishigaki, R. Gour, and J. P. Jue, "On Reducing IoT Service Delay via Fog Offloading," IEEE Internet of Things Journal, vol. 5, pp. 998-1010, January 2018. [Online]. Available: https://doi.org/10.1109/JIOT.2017.2788802.

[24] S. Zhao, Y. Yang, Z. Shao, X. Yang, H. Qian, and C.-X. Wang, "FEMOS: FogEnabled Multitier Operations Scheduling in Dynamic Wireless Networks," IEEE Internet of Things Journal, vol. 5, pp. 1169-1183, February 2018. [Online]. Available: https://doi.org/10.1109/JIOT.2018.2808280.

[25] X. Zheng, Z. Cai, J. Li, and H. Gao, "Scheduling Flows With Multiple Service Frequency Constraints," IEEE Internet of Things Journal, vol. 4, pp. 496-504, June 2016. [Online]. Available: https://doi.org/10.1109/JIOT.2016.2577630. 
[26] S. Bhandari, S. K. Sharma, and X. Wang, "Latency Minimization in Wireless IoT Using Prioritized Channel Access and Data Aggregation," in GLOBECOM 2017 2017 IEEE Global Communications Conference, December 2017. [Online]. Available: https://doi.org/10.1109/GLOCOM.2017.8255038.

[27] H. A. A. Al-Kashoash, H. M. Amer, L. Mihaylova, and A. H. Kemp, "OptimizationBased Hybrid Congestion Alleviation for 6LoWPAN Networks," IEEE Internet of Things Journal, vol. 4, pp. 2070-2081, September 2017. [Online]. Available: https://doi.org/10.1109/JIOT.2017.2754918.

[28] L. Zhao, W. Sun, Y. Shi, and J. Liu, "Optimal Placement of Cloudlets for Access Delay Minimization in SDN-Based Internet of Things Networks," IEEE Internet of Things Journal, vol. 5, pp. 1334-1344, March 2018. [Online]. Available: https://doi.org/10.1109/JIOT.2018.2811808.

[29] C. J. C. H. Watkins and P. Dayan, "Q-learning," Machine Learning, pp. 279-292, May 1992. [Online]. Available: https://link.springer.com/article/10.1007/BF00992698.

[30] V. Mnih, K. Kavukcuoglu, D. Silver, A. A. Rusu, J. Veness, M. G. Bellemare, A. Graves, M. Riedmiller, A. K. Fidjeland, G. Ostrovski, S. Petersen, C. Beattie, A. Sadik, I. Antonoglou, H. King, D. Kumaran, D. Wierstra, S. Legg, and D. Hassabis, "Humanlevel control through deep reinforcement learning," Nature, pp. 529-533, February 2015. [Online]. Available: https://www.nature.com/articles/nature14236.

[31] X. Glorot and Y. Bengio, "Understanding the difficulty of training deep feedforward neural networks," in Proceedings of the Thirteenth International Conference on Artificial Intelligence and Statistics, vol. 9, pp. 249-256, March 2010. [Online]. Available: http://proceedings.mlr.press/v9/glorot10a/glorot10a.pdf. 
[32] D. P. Kingma and J. Ba, "Adam: A Method for Stochastic Optimization," in arXiv preprint arXiv:1412.6980v9, January 2017. [Online]. Available: https://arxiv.org/abs/1412.6980.

[33] A. Sivanathan, D. Sherratt, H. H. Gharakheili, A. Radford, C. Wijenayake, A. Vishwanath, and V. Sivaraman, "Characterizing and classifying IoT traffic in smart cities and campuses," in 2017 IEEE Conference on Computer Communications Workshops (INFOCOM WKSHPS), May 2017. [Online]. Available: https://doi.org/10.1109/INFCOMW.2017.8116438.

[34] Y. Amar, H. Haddadi, R. Mortier, A. Brown, J. Colley, and A. Crabtree, "An Analysis of Home IoT Network Traffic and Behaviour," in arXiv preprint arXiv:1803.05368, March 2018. [Online]. Available: https://arxiv.org/abs/1803.05368.

[35] S. Ha, I. Rhee, and L. Xu, "CUBIC: a new TCP-friendly high-speed TCP variant," $A C M$ SIGOPS Operating Systems Review, vol. 42, pp. 64-74, July 2008. [Online]. Available: https://doi.org/10.1145/1400097.1400105.

[36] M. Mathis, N. Dukkipati, and Y. Cheng, "Proportional Rate Reduction for TCP," in Internet Engineering Task Force (IETF), RFC 6937, May 2013. [Online]. Available: https://tools.ietf.org/html/rfc6937.

[37] "ns-3." https://www.nsnam.org/. Accessed: 2018-12-12.

[38] "tiny-dnn." https://github.com/tiny-dnn/tiny-dnn. Accessed: 2018-12-12.

[39] I. Hubara, M. Courbariaux, D. Soudry, R. El-Yaniv, and Y. Bengio, "Binarized Neural Networks," in 30th Conference on Neural Information Processing Systems (NIPS 2016), December 2016. [Online]. Available: https://papers.nips.cc/paper/6573-binarizedneural-networks.pdf. 